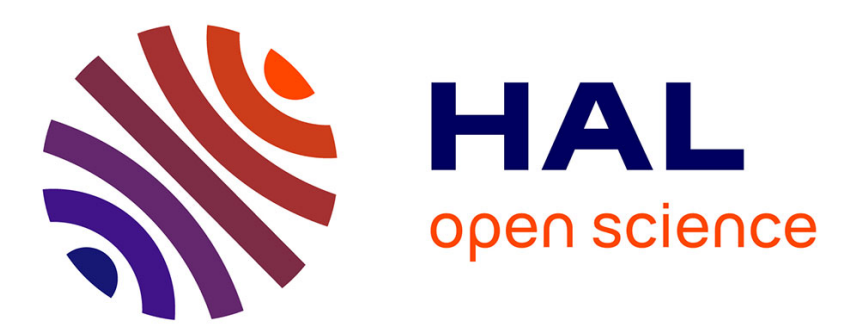

\title{
On the Behavior of MEMD in Presence of Multivariate Fractional Gaussian Noise
}

\author{
Ali Komaty, Abdel-Ouahab Boudraa, Patrick Flandrin, Pierre-Olivier
}

Amblard, Jacques-Andre Astolfi

\section{- To cite this version:}

Ali Komaty, Abdel-Ouahab Boudraa, Patrick Flandrin, Pierre-Olivier Amblard, Jacques-Andre Astolfi. On the Behavior of MEMD in Presence of Multivariate Fractional Gaussian Noise. IEEE Transactions on Signal Processing, 2021, 69, pp.2676-2688. 10.1109/TSP.2021.3074005 . hal-03372943

\section{HAL Id: hal-03372943 \\ https://hal.science/hal-03372943}

Submitted on 11 Oct 2021

HAL is a multi-disciplinary open access archive for the deposit and dissemination of scientific research documents, whether they are published or not. The documents may come from teaching and research institutions in France or abroad, or from public or private research centers.
L'archive ouverte pluridisciplinaire HAL, est destinée au dépôt et à la diffusion de documents scientifiques de niveau recherche, publiés ou non, émanant des établissements d'enseignement et de recherche français ou étrangers, des laboratoires publics ou privés. 


\title{
On the Behavior of MEMD in Presence of Multivariate Fractional Gaussian Noise
}

\author{
Ali Komaty, Abdel-Ouahab Boudraa, Senior, IEEE, \\ Patrick Flandrin, Fellow, IEEE, Pierre-Olivier Amblard, and Jacques-André Astolfi
}

\begin{abstract}
Multivariate empirical mode decomposition (MEMD) has been introduced to make standard EMD suitable for direct multichannel signals processing. Unlike EMD, MEMD is able to align sifted intrinsic mode functions (IMFs) from multiple data channels. The aim of this work is to analyze the behavior of MEMD under multivariate fGn (MfGn) with different Hurst exponents, $H$, and strengths of link between pairs of channels, $\rho$, of each sifted IMF. We report results supporting the claim that, regardless of $\rho$ values and for both MfGns long-range and short-range dependent, MEMD acts as filter bank on each channel of the input multivariate signal. Whatever the $\rho$ and $H$ values, this equivalent filter bank structure is dyadic with constant- $Q$ band-pass filters. The observed self-similar filter bank structure leads to a deeper statistical studies of the variance distribution and zero-crossings alignment in order to express this self-similarity in terms of spectral densities of multidimensional IMFs. These statistical properties generalize what was previously conducted for EMD to MEMD and estimation strategy of $H$ exponent is proposed. The filter bank behavior of MEMD is illustrated on real turbulent flow data and the estimated $H$ exponent brings out the long-range-dependent nature of the turbulent flow data. An application to EEG data is also proposed.
\end{abstract}

Index Terms-EMD, Multivariate EMD, filter banks, Multivariate fractional Gaussian noise, Hurst exponent.

\section{INTRODUCTION}

Empirical mode decomposition (EMD) is a fully data-driven method to decompose real world multicomponent signals into a reduced number of intrinsic mode functions (IMFs), which are typically AM-FM signals from which meaningful instantaneous amplitude and frequency are estimated [1],[2],[3],[4]. Properties of this decomposition, such as locality, completeness, data-driven nature and multi-resolution aspect, have made EMD a valuable tool for real world non-stationary signals analysis and particularly in deterministic situations [5],[6],[7],[8],[9],[10],[11]. For stochastic situations involving broadband noise, namely, fractional Gaussian noise (fGn), EMD acts as dyadic filter bank [12]. Despite its striking features, EMD is only designed for univariate data. For signals containing data channels (multivariate), application of EMD channel-wise is suboptimal because it fails to effectively align frequency responses from same index of IMFs of multiple channels, and this makes IMF-by-IMF comparisons infeasible

A. Komaty is with USAL, Beirut, Lebanon (akomaty@gmail.com). A.O. Boudraa and J.A. Astolfi are with IRENav, Ecole Navale/ENSAM, Brest, France ((astolfi,boudra)@ecole-navale.fr). P. Flandrin is with ENS de Lyon, Laboratoire de Physique, France (Patrick.Flandrin@ens-lyon.fr). P.O. Amblard is with GiPSA-Lab, Université de Grenoble Alpes, France. (pierreolivier.amblard@gipsa-lab.grenoble-inp.fr).
[13]. To resolve this problem, multivariate extension of EMD (MEMD) has been designed to process a general class of multivariate signals with an arbitrary number of channels, and this is also motivated by recent advances in sensors and data acquisition tools [14]. The aim of MEMD is to give more insight into dynamics and interdependence between multiple channels of the analyzed signal. This decomposition acts as a quasi-dyadic filter bank under white Gaussian noise (wGn) [15], and symmetric $\alpha$-stable noise [16]. Scale-aligned modes extracted by MEMD give better defined sub-band filters compared to EMD [17]. It has been verified that under fGn, the dyadic filter bank property of MEMD is preserved [18][19]. However, these results have been obtained assuming that components of multichannel data are uncorrelated, while in many fields of applications multivariate measurements involve specific properties such as short or long-range dependence of the channels and self-similarity. To best of our knowledge, up to now much less attention has been paid to the behavior of MEMD for multivariate fGn (MfGn) as well as its exploitation for Hurst exponent estimation. For more realistic situations, it is important to investigate whether the filter bank structure is preserved by MEMD under MfGn with correlated channels. This work generalizes the studies conducted under wGn [15], fGn [19], MfGn with uncorrelated channels [18],[20], and also the findings in the case of EMD [12],[21].

\section{Main contributions}

1) We provide, to the best of our knowledge, the first behavior analysis of MEMD under MfGn for different Hurst exponents, $H$, and strengths of link, $\rho$, between pairs of channels of each sifted IMF. The analyzed statistical properties allows us to generalize what was previously shown for EMD to MEMD.

2) We show that whatever $H$ and $\rho$ values, MEMD acts as filter bank on each channel of the input signal, and this structure is dyadic with constant- $Q$ band-pass filters. Regardless of $\rho$ values, and for both MfGns long-range dependent and short-range dependent, first IMF presents the characteristics of a high-pass filter, while the other IMFs behave similarly to a band-pass filter.

3) Using the filter bank structure of MEMD, we get access to the Hurst exponent $H$ via the variance progression across IMFs. We report results showing that for low and high correlated channels, MEMD-based estimator performs similarly than the discrete wavelet transform-based estimator, but with the lowest standard deviations. 
4) We highlight the fact that for correlated channels, the sifted modes are also correlated and the intensity of their link is related to the Hurst exponents of these channels.

5) Filter bank behavior is illustrated on real turbulent flow data and estimated $H$ exponent brings out long-range-dependent nature of turbulent flow data. Different power law behaviors are observed on spectra of the modes, and particularly Kolmogorov's spectral slope.

The paper is organized as follows. In section II we point out the interest of multichannel data and we stress the importance of taking into account the interdependence between channels for processing of these data. Basics of EMD and MEMD are given in Section III. We detail the MfGn model in Section IV. Analyzed properties of MEMD and simulation setup are presented in Section V. Results are presented and analyzed in Section VI. Finally, conclusion is given in Section VII.

\section{Multichannel DATA}

Advances in sensor and data recording technologies has resulted in the production of multichannel sets of data [22]. These new generations of sensors produce signals containing several data channels. Consequently, multivariate time series analysis has gained popularity, particularly, in applications involving several recording of signals from a single system. A growing number of signal applications requiring multichannel data processing, include biomedical processing (EEG/MEG/fMRI), color image processing and array processing. Components of multichannel signal may have different scales, and particularly they may be correlated. Thus, processing of such signals channel-wise is suboptimal since the interdependence (correlation) between channels of these signals is not exploitable and thus, hidden features of the system (brain...) cannot be revealed. Tools such as MEMD are expected to give more insight into the dynamics and the interdependence between channels of such signals. Furthermore, there is an increase interest and significant research effort into understanding real/complex biological or physical networks. Indeed, many systems are considered as systems where the global behavior emerges from the interaction and cannot be predicted from the sole observation of the individuals [23].

\section{BASICS OF EMD AND MEMD}

Unlike standard decompositions that project data onto predefined basis functions, bases of EMD and MEMD are derived from the data. While EMD decomposes univariate data into slow and fast oscillations, MEMD expands multivariate data into slow and fast generalized oscillations (rotations).

\section{A. EMD}

EMD expands any real-valued signal $x(t)$ into a limited number of IMFs. We refer to discrete version of $x(t)$ by index sequences: $x[n], n \in\{1,2, \ldots, N\}$. Being fully data driven, IMFs represent the inherent temporal modes (scales) that characterizes $x[n]$. These modes are required to be narrowband. By construction, each IMF is a zero-mean whose number of zero-crossings differs at most by one from the number of its extrema. EMD ends up with an expansion of the form:

$$
x[n]=\sum_{m=1}^{M_{c}} c_{m}[n]+d[n]
$$

where $M_{c}$ is the number of IMFs and $c_{m}[n]$ denotes the $m^{t h}$ IMF. Modes, $\left(c_{m}[n]\right)_{m=1}^{M_{c}}$, in equation (1) are the bases of $x[n]$, and are sparse (with $M_{c} \ll N$ ) and template free. The component $d[n]$ is called the residual of the decomposition and cannot contain a full oscillation [1]. Designed to operate in time-domain, EMD has been extended to handle complex-valued time-series [24],[25],[26]. Complex version introduced by Tanaka and Mandic [24] is based on the inherent relationship between positive and negative frequency components of a complex-valued signal, that are treated as two separate independent signals. Since frequency components are generally mutually dependent, this can lead to a loss of information in the signal. To overcome this limitation, Altaf et al., [25] developed an alternative version, that operates completely in $\mathbb{C}$ domain, and sifts a single set of complex IMFs. Another extension, introduced by Rilling et al., called bivariate EMD [26], separates "fast rotating" components of a complex-valued signal from "slowly rotating" ones. Envelope curves are obtained by projecting input signal in multiple directions. Local mean is calculated by averaging envelope curves and is then subtracted from input signal repeatedly to sift out rotating components [26]. To tackle multidimensional signal problem, EMD has first extended to trivariate signals where a triviate signal is represented as a pure quaternion, with each of its component a real-valued time series [27]. All these extensions have led to the development of MEMD.

\section{B. $M E M D$}

MEMD is an extension of EMD developed to process multivariate data having an arbitrary number of input channels [14]. These data exhibit rotational components that require appropriate treatment for their time-frequency analysis. Thus, principle of separating oscillations that underpins EMD is generalized for separating rotations. An important property of MEMD is the modes alignment which consists in finding a set of common scale/modes across different components of a multivariate signal, thus ensuring that the modes are matched both in number and in scale properties [14]. Consider a sequence of $P$-dimensional vectors $\mathbf{x}(t)=\left(x_{1}(t), x_{2}(t), \ldots, x_{P}(t)\right)_{t \in \mathbb{R}}$ representing a multivariate signal. We refer to discrete version of $\mathbf{x}(t)$ by sequence: $\mathbf{x}[n]=\left(x_{1}[n], x_{2}[n], \ldots, x_{P}[n]\right)$ where $n \in\{1,2, \ldots, N\}$. MEMD uses a vector-value form of relation (1) to decompose the $P$-variate signal $\mathbf{x}[n]$ as follows

$$
\mathbf{x}[n]=\sum_{m=1}^{M} \mathbf{c}_{m}[n]+\mathbf{d}[n], \mathbf{c}_{m}, \mathbf{d} \in \mathbb{R}^{P}
$$

where $M$ is the number of extracted $P$-variate modes, $\left(\mathbf{c}_{m}\right)_{m=1}^{M}\left(\mathbf{c}_{m}[n]=\left(c_{m, 1}[n], \ldots, c_{m, P}[n]\right)\right)$, contains scalealigned intrinsic joint rotational modes and $\mathbf{d}[n]$ is the $P$ variate residue. Expansion generated by MEMD is a sparse decomposition (with $M \ll N$ ). MEMD estimates the localmean, in a $P$-dimensional space through averaging of multiple 
envelopes obtained by taking signal projection along multiple uniformly distributed directions in this space. Extrema of such univariate projections are then interpolated component-wise and averaged to yield multi-dimensional signal mean [14]. To obtain the projections of $\mathbf{x}[n]$ in $P$-dimensional space, a sampling scheme based on low discrepancy Hammersley sequence is used [28]. The direction vectors are governed by an approximate sampling of a $P$-dimensional hypersphere and accuracy of mean calculation depends on the distribution of direction vectors. Let us consider $\mathbf{s}_{\theta v}=\left(s_{1}^{v}, s_{2}^{v}, \ldots, s_{P}^{v}\right)_{v=1}^{V}$ a set of direction vectors along directions given by angles $\theta_{\mathbf{v}}=\left(\theta_{v 1}, \theta_{v 2}, \ldots, \theta_{v P-1}\right)$. Algorithm 1 summarizes the different steps of MEMD where an uniform sampling scheme is used to assign equal weighting to all the projection directions. A noise-assisted version of MEMD (NA-MEMD) has been proposed for computation of EMD, in order to produce localized frequency estimates at accuracy level of instantaneous frequency [29]. Reported results show the interest of NAMEMD applied channel-wise, over EMD and EEMD.

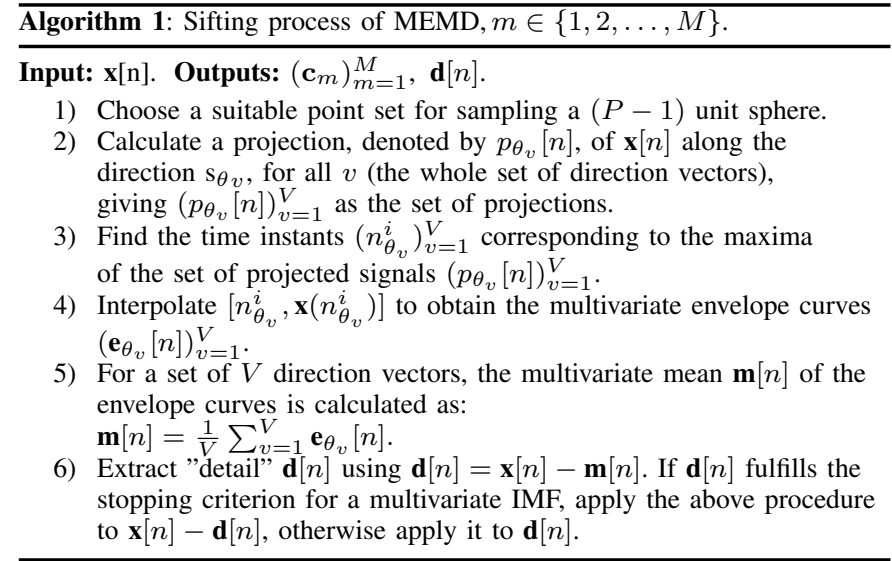

\section{Multivariate FGn Model}

fGn is of a particular interest in signal processing for its ability to describe long-range dependent phenomena. This noise is defined as an approximate derivative of the fractional Brownian motion (fBm), which is a self-similar centered continuous Gaussian process. Discrete fGn is a discrete-time process defined as the increment process of the $\mathrm{fBm}$, which is a self-similar Gaussian process with stationary increments [30]. The increment process may exhibit long-range dependence, and is commonly used in modeling physical and biological phenomena [31]-[32]. However, in many fields of applications such as in physics, neurosciences or network traffic, multivariate measurements are performed and they involve salient properties such as short/long-range dependence, fractality and self-similarity [33]. To deal with such multivariate, $\mathrm{fBm}$ has been extended to multivariate framework (MfBm) [23],[34]. MfBm is a Gaussian multivariate signal whose components are correlated scalar fBm with different Hurst exponents satisfying three properties, namely Gaussianity, self-similarity and stationarity of the increments. Here, self-similarity has to be understood as joint-similar. Let $\mathbf{y}(t)=\left(y_{1}(t), \ldots, y_{P}(t)\right)_{t \in \mathbb{R}}$ be a $P$-dimensional $\mathrm{MfBm}$ having components jointly-similar parametrized by $P$ different Hurst exponents $H=\left(H_{1}, \ldots, H_{P}\right) \in[0,1]^{P}, P$ scaling coefficients $\sigma_{i}=\sqrt{\operatorname{var}\left(y_{i}(1)\right)}$ (standard deviation of $i^{\text {th }}$ component at $t=1$ ) and also, by $P^{2}$ real correlation coefficients $\rho_{i j}=\operatorname{corr}\left(y_{i}(1), y_{j}(1)\right) \in[-1,1]$ between the components $y_{i}(t)$ and $y_{j}(t)$ at $t=1$ with $\rho_{i j}=\rho_{j i}$ allowing these components to be more or less strongly correlated (for $i, j=1, \ldots, P$ with $j>i$ ) [23]. Each component $\left(y_{i}(t)\right)_{t \in \mathbb{R}}$ is an univariate $\mathrm{fBm}$. The multivariate process is also characterized by an antisymmetric matrix whose elements are $\eta_{i j}$ and controls the time asymmetry of $\mathbf{y}(t), \eta_{i j}=-\eta_{j i}$. If the process is time-reversible, parameters $\eta_{i j}$ are all equal to zero and if the process admits a causal representation, $\eta_{i j}$ values are function of $\rho_{i j}, H_{i}$ and $H_{j}$. Two natural cases are considered, namely causal $\mathrm{MfBm}$ and well-balance $\mathrm{MfBm}$. As for $\mathrm{fBm}, \mathrm{MfBm}$ has by definition stationary increments, and a covariance structure of the increments process. The first difference of the MBfm process $\mathbf{x}(t)=\Delta \mathbf{y}(t)=\mathbf{y}(t+1)-\mathbf{y}(t)$ is a MfGn with correlated components [23]. From covariance function of $\mathbf{y}(t)$, we deduce the covariance of $\mathbf{x}(t)$. Structure of the MfGn defined by the cross-covariance of the increments of the components $y_{i}(t)$ and $y_{j}(t)$ of $\mathbf{y}(t)$ is given by

$$
\gamma_{i j}(h)=\mathbb{E}\left[x_{i}(t) x_{j}(t+h)\right]
$$

In the sequel, let $|h| \geq 1$. For all $(i, j) \in\{1, \ldots, P\}^{2}, i \neq j$, cross-covariance of $x_{i}(t)$ and $x_{j}(t)$ is given by

$$
\gamma_{i j}(h)=\frac{\sigma_{i} \sigma_{j}}{2}\left[w_{i j}(h-1)-2 w_{i j}(h)+w_{i j}(h+1)\right]
$$

The function $w_{i j}(h)$ is defined by [33]

$$
w_{i j}(h)= \begin{cases}{\left[\rho_{i j}-\eta_{i j} \operatorname{sign}(h)\right]|h|^{H_{i}+H_{j}}} & \text { if } H_{i}+H_{j} \neq 1 \\ \rho_{i j}|h|+\eta_{i j} h \log |h| & \text { if } H_{i}+H_{j}=1\end{cases}
$$

where $\eta_{i j}$ is given by

1) Causal case:

$\eta_{i j}=\left\{\begin{array}{lr}-\rho_{i j} \tan \left[\frac{\pi}{2}\left(H_{i}+H_{j}\right)\right] \tan \left[\frac{\pi}{2}\left(H_{i}-H_{j}\right)\right] & \text { if } H_{i}+H_{j} \neq 1 \\ \rho_{i j} \frac{2}{\pi \tan \left(\pi H_{i}\right)} & \text { if } H_{i}+H_{j}=1\end{array}\right.$

2) Well-balanced case: $\eta_{i j}=0$.

\section{SOME PROPERTIES OF MEMD UNDER MFGN}

To shed light on the properties of MEMD, its performances are analyzed under MfGn. The aim is to bring some properties around the filter bank concept. However, as it has been stressed in [15] the idea of filter bank structure for multivariate signals is ambiguous, since the concept of frequency is not clearly defined for multivariate signals. Thus, we consider the frequency response for individual channels and we investigate channel-wise whether the filter bank property is preserved with correlated channels. We also investigate if this filter bank structure is dyadic with constant-Q band pass filters or not. We use the correlogram to analyze the intensity of coupling between pairs of channels of each sifted IMF and its link to Hurst component. Finally, we study if the hierarchy of the sifted IMFs can be exploited to get access to Hurst exponents. 


\section{Simulation SET-UP}

Extensive simulations are carried out on MfGn processes generated using the method developed in [33]. We have generated $J=5000$ snapshots of 4 -channel fGn $(P=4)$ with an identical length of $N=1000$ data points, and for different $H$ values and strengths of link between pairs of channels of MfGn. Even a complete study has been conducted within ranges $0.1 \leq H \leq 1$ and $0 \leq \rho \leq 1$, only four typical values of $H$ and $\rho$, are displayed for the sake of readability. We limit ourself to causal case (Eq. 6). All the components are equally correlated with parameter $\rho_{i j}=\rho \in\{0,0.2,0.5,0.8\}$. MfGn has components jointly-similar parametrized by $P$ Hurst values $H \in\{0.2,0.4,0.6,0.8\}$. Notations of these simulations are expressed as follows:

1) $x_{H_{p}}^{(j)}[n]$ is the $j^{t h}$ simulated $p^{\text {th }}$ fGn path of length $N$ where $n \in\{1,2, \ldots, N\}, p \in\{1,2, \ldots, P\}$ and $j \in\{1,2, \ldots, J\}$.

2) $c_{m, H_{p}}^{(j)}[n]$ is the $p^{t h}$-dimension of the $m^{t h}$ IMF of parameter $H_{p}$ sifted by MEMD of the $j^{t h}$ simulated $P$-variate fGn where $m \in\{1,2, \ldots, M\}$. We use standard EMD for comparison, which is applied channel-wise, and even though the number of IMFs will not be equal to $M$, we will restrict our study to $M=8 P$-variate IMFs.

\section{RESULTS}

We report simulations on synthetic and real world data to illustrate the properties and the behavior of MEMD. Conclusions are drawn for different Hurst exponents and correlation values between pairs of channels of each sifted mode. An application to EEG data is also presented.

\section{A. MEMD equivalent filter banks}

Spectral analysis of a MfGn time series is carried out for each sifted IMF using an estimate of the PSD given by:

$\hat{S}_{m, H_{p}}(f)=\sum_{\tau=-N+1}^{N-1} \hat{r}_{m, H_{p}}[\tau] w[\tau] \exp (-i 2 \pi f \tau), \quad|f|<0.5$

where $w[\tau]$ is the Hamming window, and

$$
\hat{r}_{m, H_{p}}[\tau]=\frac{1}{J} \sum_{j=1}^{J}\left(\frac{1}{N} \sum_{n=1}^{N-|\tau|} c_{m, H_{p}}^{(j)}[n] c_{m, H_{p}}^{(j)}[n+|\tau|]\right)
$$

is the ensemble average over $J$ realizations of the empirical estimates of the auto-correlation function of IMF $c_{m, H_{p}}^{(j)}[n]$, where $|\tau| \leq(N-1)$. Results of this analysis plotted in figures 1 and 2 where PSDs of sifted IMFs are estimated for different combinations of $(\rho, H)$, and results are means over $J$ Monte Carlo runs. Graphs plotted in these figures reveal striking properties. Overlapping of the frequency bands corresponds to the same-index IMFs, showing the mode alignment. Despite some leaks observed for more fractured and correlated channels, scaled-aligned IMFs give well defined sub-band filters. These results show that leaks do not occur for non-coupled channels, and suggest that leaks appear for low coupled and more fractured channels. This set of filters tend to organize in filter bank structure similar to wavelet decompositions. Regardless of $\rho$ values, and for both MfGns long-range dependent and short-range dependent, graphs of figures 1 and 2 reveal that the behavior of the first IMF contrasts with that of the other IMFs in the sense that the first IMF presents the characteristics of a high-pass filter, while IMFs of higher indexes $(m \geq 2)$ behave similarly to a band-pass filter. Observe that for $H \in[0.2,0.8]$ spectra of IMFs for $m \in\{2,3, \ldots, 8\}$, look quite similar except the shifts in frequency and scaling in amplitude. Also, theses figures show that the energy balance between IMFs reflects quite well the behavior of the theoretical spectrum (superimposed dashed-dotted curve). As evidenced, decreasing (increasing) power-law spectrum is observed when $H>0.5$ $(H<0.5)$. For $H=0.5$, flat spectrum corresponds to $\mathrm{wGn}^{\mathrm{G}}$ (Fig. 3). All these results confirm (and is a generalization of) the findings of Flandrin et al. for EMD [12].
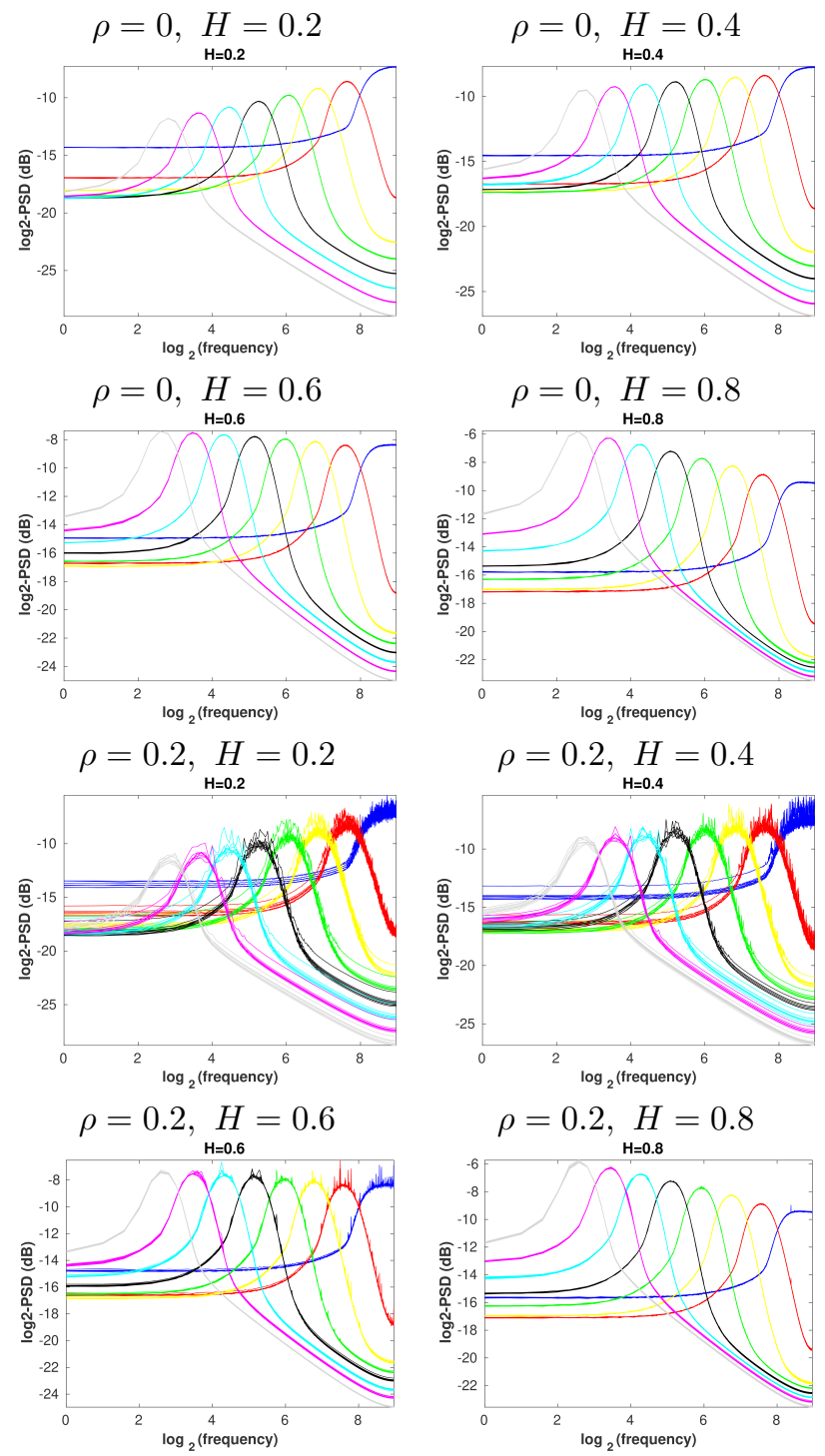

Fig. 1. PSDs of IMFs for $H \in\{0.2,0.4,0.6,0.8\}$ and $\rho \in\{0,0.2\}$.

\section{B. Filter bank structure}

A salient property of an IMF is that it can be fully described by its extrema and this can be exploited for different 

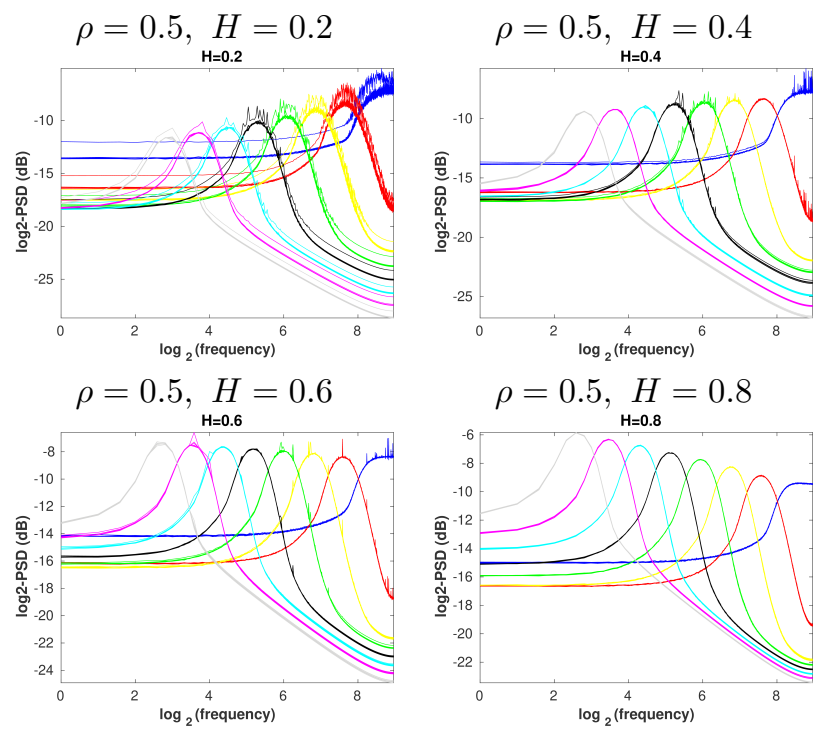

$\rho=0.8, \underset{H=02}{H}=0.2$
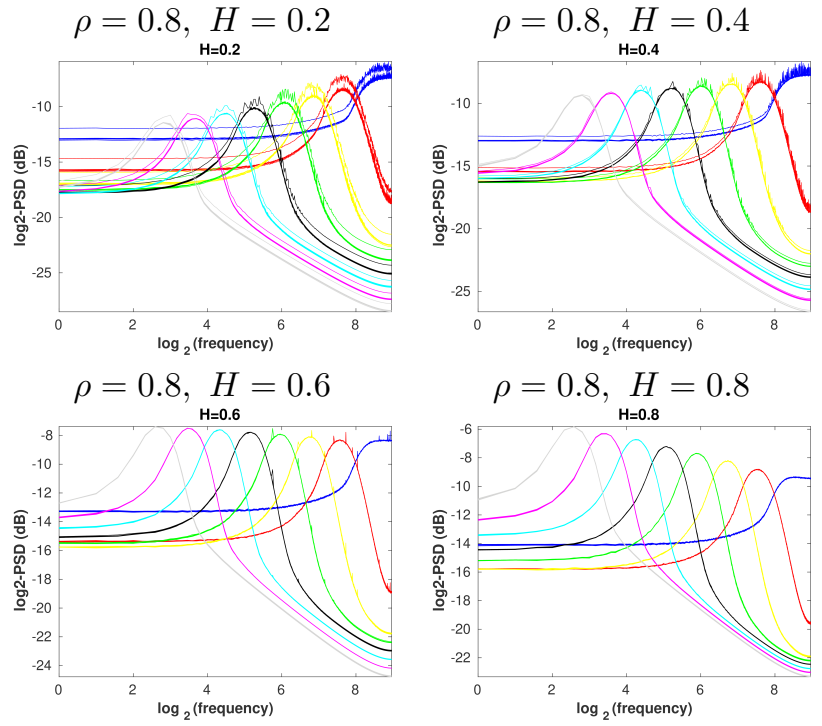

Fig. 2. PSDs of IMFs for $H \in\{0.2,0.4,0.6,0.8\}$ and $\rho \in\{0.5,0.8\}$.

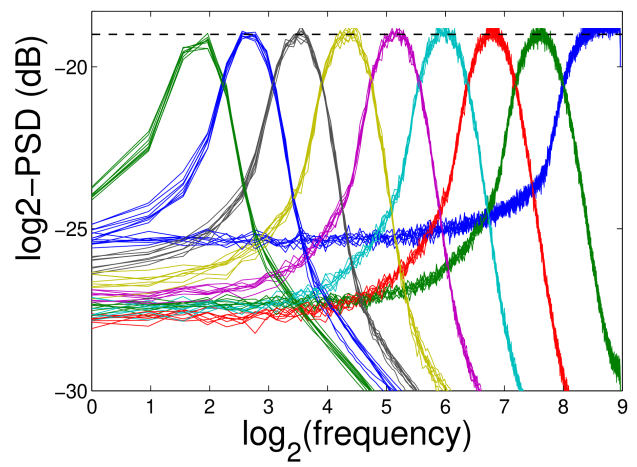

Fig. 3. PSD of IMFs for $H=0.5$ (wGn case). applications such as frequency analysis [1], image and signals coding [35],[36], or signals watermarking [37]. Thus, filter bank structure is quantitatively appreciated by measuring the frequency content of each IMF using its extrema, and more precisely its zero-crossings (ZCs). Measuring average number of ZCs, $z_{H_{p}}[m]$, of an IMF is a meaningful way of characterizing its mean frequency, and the way this varies from IMF to IMF is an indication of hierarchical structure of the equivalent filter bank [12]. Graphical representation of the logarithm of $z_{H_{p}}[m]$ for selected values of $\rho$ and $H$ is plotted in figure 4 where each straight line has a slope less than -0.9 (close to -1 ), indicating an almost dyadic decrease across the modes. These results evidence that whatever $\rho$ and $H$ values, MEMD acts under MfGn as a filter bank. Up to slight dependences on $\rho$ and $H$, we observe that $z_{H_{p}}[m]$ is divided by 2 when going from IMF to the next one. Theses results are in agreement and generalize findings reported for wGn [15] and for fGn [19].

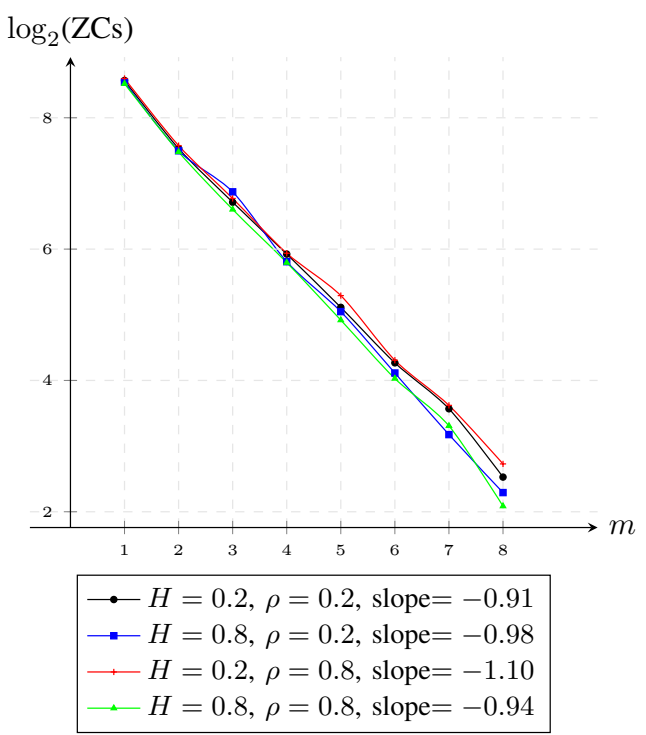

Fig. 4. Comparison of logarithm of $z_{H_{p}}[m]$ for selected values of $\rho$ and $H$.

\section{Constant- $Q$ band pass filters}

As evidenced in figure 4 , ZCs $z_{H_{p}}[m]$ is a decreasing exponential function $m$, that can be modeled by the relation

$$
z_{H_{p}}[m] \propto \beta_{H_{p}}^{-m}
$$

where scaling factor $\beta_{H_{p}}$ is the average decrease rate of ZCs, estimated using least square (LS) fitting of a semi-log diagram of $\log _{2}\left(z_{H_{p}}[m]\right)$ vs $m \in[1,8]$. Since each straight line has a slope close to -1 (Fig. 4), thus we get

$$
\begin{aligned}
& \log _{2}\left(z_{H_{p}}[m+1]\right)-\log _{2}\left(z_{H_{p}}[m]\right) \approx-1 \\
& \quad \log _{2}\left(\frac{\beta_{H_{p}}^{-(m+1)}}{\beta_{H_{p}}^{-m}}\right)=\log _{2}\left(\beta_{H_{p}}^{-1}\right) \approx-1 \Rightarrow \beta_{H_{p}} \approx 2
\end{aligned}
$$

The decreased in the number of ZCs as $m$ increases, can be approximated by the quadratic expression

$$
\beta_{H_{p}} \approx 2+\delta_{1}\left(H_{p}-0.5\right)-\delta_{2}\left(H_{p}-0.5\right)^{2}
$$


where $\delta_{1}=0.06$ and $\delta_{2}=0.02$. Accuracy of this approximation depends on the way to sift the IMFs and, particularly on stopping criterion and uniformity of sampling, in the $P$ dimensional space, used (Step 1 of Algorithm 1). For EMD, the decreased in the number of ZCs is given by [38]:

$$
\beta_{H_{p}} \approx 2.01+0.2\left(H_{p}-0.5\right)+0.12\left(H_{p}-0.5\right)^{2}
$$

Since $z_{H_{p}}[m]$ is divided by 2 when going from one IMF to the next one, we can check for a possible self-similarity in the filter-bank structures (Figs. 1-2). If we restrict ourselves to the band-pass modes ( $m=2$ to $m=8$ ), self-similarity is traduced by the scale relation

$$
S_{l, H_{p}}(f)=\beta_{H_{p}}^{a_{p}(l-m)} S_{m, H_{p}}\left(\beta_{H_{p}}^{a_{p}(l-m)} f\right)
$$

for some $a_{p}$ and $l>m \geq 2$. Thus, PSDs of IMFs, should collapse onto a single curve when properly renormalized. This is illustrated in Figs. 5-6, with $a_{p}=2 H_{p}-1$ and $\beta_{H_{p}}=2$. Overall, this setting verifies this assumption, as the corresponding renormalization converge to the same template. Regardless $\rho$ and $H$ values, normalized PSDs collapse onto a single curve showing self-similarity of the band-pass filters. More precisely, IMFs of individual channels exhibit self-similar behavior. However, we note the presence of discrepancies in low frequency domain for $\rho=0$ and for $H \geq 0.4$. Overall, diagrams of figures 5 and 6 support the idea that MEMD acts as a dyadic filter bank of constant-Q band-pass filters. These results are in agreement with the findings reported in [12] in the case of EMD, and with [15] for wGn case $(H=0.5)$.

\section{MEMD-based estimation of Hurst exponent}

The filter bank behavior of MEMD suggests to investigate its potential to get access to Hurst exponents. To this end, the variance progression across the modes to estimate $H_{p}$ value is exploited. Given the self-similar relation (13) of band-pass IMFs $(m \geq 2)$, one can deduce how variance should evolve as a function of $m$ [39]. Assuming that relation (13) holds for any $a_{p}=2 H_{p}-1$ and $l>m \geq 2$, variance, $V_{H_{p}}[l]$, of the $p^{\text {th }}$-dimensional component of the $l^{\text {th }}$ mode is given by

$$
V_{H_{p}}[l]=\operatorname{var}\left(c_{l, H_{p}}[n]\right)=\int_{-1 / 2}^{1 / 2} S_{l, H_{p}}(f) d f
$$

Inserting relation (13) in (14), for fixed $m$ we get

$$
\begin{aligned}
V_{H_{p}}[l] & =\beta_{H_{p}}^{a_{p}(l-m)} \int_{-1 / 2}^{1 / 2} S_{m, H_{p}}\left(\beta_{H_{p}}^{(l-m)} f\right) d f \\
& =\beta_{H_{p}}^{2\left(H_{p}-1\right)(l-m)} V_{H_{p}}[m]
\end{aligned}
$$

For fixed $l$ we get

$$
V_{H_{p}}[m]=\beta_{H_{p}}^{2\left(H_{p}-1\right) m} V_{H_{p}}[l] \beta_{H_{p}}^{-2\left(H_{p}-1\right) l}
$$

Setting $l=2$ in relation (16), we get

$$
V_{H_{p}}[m]=\beta_{H_{p}}^{2\left(H_{p}-1\right) m} \underbrace{V_{H_{p}}[2] \beta_{H_{p}}^{4\left(H_{p}-1\right)}}_{A_{H_{p}}}
$$

which leads to relation

$$
V_{H_{p}}[m]=A_{H_{p}} \beta_{H_{p}}^{2\left(H_{p}-1\right) m}
$$

where $V_{H_{p}}[2]$ is estimated using relation (26). As a consequence of self-similar structure of the decomposition, using relation (13) we derive the relation between the mean periods of IMFs, $\bar{T}_{H_{p}}[$.$] , for l>m \geq 2$ as follows

$$
\bar{T}_{H_{p}}[l]=\beta_{H_{p}}^{(l-m)} \bar{T}_{H_{p}}[m]
$$

where the estimated mean period, based on $\mathrm{ZCs}$, is given by

$$
\hat{\bar{T}}_{H_{p}}[m]=\frac{\text { Distance between the first and the last ZC }}{\text { Number of ZCs-1 }}
$$

Setting $l=2$ into relation (19) we get

$$
\beta_{H_{p}}^{(2-m)}=\frac{\bar{T}_{H_{p}}[2]}{\bar{T}_{H_{p}}[m]} \Rightarrow \beta_{H_{p}}^{2 m H_{p}}=\frac{\left(\bar{T}_{H_{p}}[2]\right)^{-2 H_{p}} \beta_{H_{p}}^{4 H_{p}}}{\left(\bar{T}_{H_{p}}[m]\right)^{-2 H_{p}}}
$$

It follows from relation (18) that

$$
V_{H_{p}}[m]=\frac{A_{H_{p}}\left(\bar{T}_{H_{p}}[2]\right)^{2}}{\beta_{H_{p}}^{4}}\left(\bar{T}_{H_{p}}[m]\right)^{-2} \beta_{H_{p}}^{2 m H_{p}}
$$

Inserting $\beta_{H_{p}}^{2 m H_{p}}$ into relation (22) one gets

$$
V_{H_{p}}[m]=D_{H_{p}}\left(\bar{T}_{H_{p}}[m]\right)^{2\left(H_{p}-1\right)}
$$

where $D_{H_{p}}$ is given by

$$
D_{H_{p}}=V_{H_{p}}[2] \beta_{H_{p}}^{8\left(H_{p}-1\right)}\left(\bar{T}_{H_{p}}[2]\right)^{2\left(1-2 H_{p}\right)}
$$

The mode variance is exponentially decreasing function of $m$, with a decay rate is a linear function of $H_{p}$ (log-log plot). Inserting $\beta_{H_{p}} \approx 2$ in (23) and taking the logarithm gives

$$
\begin{aligned}
\log _{2}\left(V_{H_{p}}[m]\right) & \approx \log _{2}\left(V_{H_{p}}[2]\right)+2\left(1-H_{p}\right)\left[\log _{2}\left(\bar{T}_{H_{p}}[2]\right)-4\right] \\
& +2\left(H_{p}-1\right) \log _{2}\left(\bar{T}_{H_{p}}[m]\right)
\end{aligned}
$$

An experimental evidence for this behavior (Eq. 25) is reported in figure 7 for different $\rho$ and $H$ values, where the (energybased) empirical variance estimate is given by:

$$
\hat{V}_{H_{p}}[m]=\frac{1}{J} \sum_{j=1}^{J}\left[\frac{1}{N} \sum_{n=1}^{N}\left(c_{m, H_{p}}^{(j)}[n]\right)^{2}\right]
$$

In this figure, the grouped blue and red dots from the upper left to the lower right represent the mean energy density (estimation of the variance) as a function of the spectrum mean period for IMFs $1-7$ over $J$ realizations. Solid black line cutting through the clouds is the weighted linear fit within IMF indexes range $m=1$ to $m=7$. For sake of clarity, all curves have been shifted upwards to avoid overlapping. As evidence in figure 7 , irrespective of intensity of channels link, the predicted relationship (18) only holds for IMF index $m>1$ (band-pass filters). Furthermore, the model of relation (25) fits well the data for $H>0.2$ with low discrepancies. A visual examination of these results shows that, the modeenergy spread is more concentrated in a more uniform clouds, yielding a better aligned IMF indexes and almost with no mode mixing effect. Using a logarithm (base 2) linearized version of relation (18), straight lines can be fitted to curves corresponding to different pairs of $(\rho, H)$, with slope $\kappa_{H_{p}}$ that gives an estimated of $H, H_{p}$. Thus, inserting $\beta_{H_{p}} \approx 2$ in relation (18) and taking the logarithm (base 2) gives

$$
\log _{2}\left(V_{H_{p}}[m]\right) \approx \log _{2}\left(2^{2\left(H_{p}-1\right) m}\right)+\log _{2}\left(A_{H_{p}}\right)
$$




$$
\approx \kappa_{H_{p}} m+\log _{2}\left(A_{H_{p}}\right)
$$

From relation (27), one can estimate $H_{p}$ by

$$
\hat{H}_{p}=1+\frac{\kappa_{H_{p}}}{2}
$$

An experimental evidence of relation (27) is given in figure 8, where values of $\log _{2}\left(V_{H_{p}}[m]\right)$ are plotted against $m$ for different values of $(\rho, H)$. Values of empirical variance estimates are represented by red dotted lines for different $H$ values. The error bars correspond to the standard deviation calculated over $J$ realizations. The black thin line is the weighted linear fit within the IMF indexes range from $m=1$ to $m=7$. Same conclusions as in figure 7 can be drawn. In these diagrams, straight line can be fitted to all curves, regardless of $\rho$ values, for $m>1$ in accordance with relation (18). Values of $\hat{H}_{p}$ are calculated using three estimators namely, MEMD, EMD and discrete wavelet transform (DWT) [40]. The same range of scales for these three estimators is exploited. For DWTestimator, "Daubechies 4" mother wavelet is used. Due to the dyadic filter bank structure of EMD and MEMD, IMFs indexes are equivalent to scales indexes. Performances of these estimators based on scales/modes $m=2$ to $m=8$ are reported in Tables I and II. The $\rho$ value is fixed, and for all $H$ values, we estimated the expected value, the standard deviation and the bias of each estimator. Estimates in Table I show that, for low and high correlated channels, MEMD-based estimator performs similarly than DWT-based estimator, but with the lowest standard deviations. MEMD-based estimator outperforms EMD-based estimator both in terms of estimate and standard deviation. Overall, whatever $\rho$ values, estimation model with slope $\kappa_{H_{p}}$ (Eq. 28) provides the best results for $H>0.2$. Recall that simulations have been carried out over 5000 realizations, thus our conclusion is valid for this range and of course, all these methods will have a better standard deviation as the number of realizations increases. Results of Table II show that MEMD-based estimator achieves the lowest bias than the two other estimators for all analyzed $H$ values.

TABLE I

EXPECTED VALUES AND STANDARD DEVIATIONS (MEAN \pm STD) OF THREE DIFFERENT ESTIMATORS FOR SELECTED PAIRS $(\rho, H)$ VALUES. BEST RESULTS ARE HIGHLIGHTED IN BOLD.

\begin{tabular}{|c|c|c|c|c|}
\hline & & \multicolumn{3}{|c|}{ Estimators } \\
\cline { 3 - 5 }$\rho$ & $\mathrm{H}$ & DWT & EMD & MEMD \\
\hline \hline \multirow{4}{*}{0} & 0.2 & $\mathbf{0 . 1 6} \pm 0.103$ & $0.28 \pm 0.083$ & $0.15 \pm \mathbf{0 . 0 6 8}$ \\
\cline { 2 - 5 } & 0.4 & $\mathbf{0 . 4} \pm 0.123$ & $0.42 \pm 0.085$ & $\mathbf{0 . 4} \pm \mathbf{0 . 0 6 9}$ \\
\cline { 2 - 5 } & 0.6 & $\mathbf{0 . 6} \pm 0.117$ & $0.61 \pm 0.082$ & $\mathbf{0 . 6} \pm \mathbf{0 . 0 6 4}$ \\
\cline { 2 - 5 } & 0.8 & $\mathbf{0 . 8} \pm 0.117$ & $0.8201 \pm 0.087$ & $0.81 \pm \mathbf{0 . 0 6 4}$ \\
\hline \hline \multirow{4}{*}{0.2} & 0.2 & $\mathbf{0 . 1 4} \pm 0.165$ & $0.11 \pm 0.112$ & $0.12 \pm \mathbf{0 . 1 0 5}$ \\
\cline { 2 - 5 } & 0.4 & $0.35 \pm 0.207$ & $0.35 \pm 0.108$ & $\mathbf{0 . 3 6} \pm \mathbf{0 . 1 0 5}$ \\
\cline { 2 - 5 } & 0.6 & $0.62 \pm 0.237$ & $0.58 \pm 0.1$ & $\mathbf{0 . 6} \pm \mathbf{0 . 0 9}$ \\
\cline { 2 - 5 } 0.8 & $0.8 \pm 0.231$ & $0.82 \pm 0.102$ & $\mathbf{0 . 8} \pm \mathbf{0 . 0 9 3}$ \\
\hline \hline \multirow{4}{*}{0.8} & 0.2 & $0.13 \pm 0.161$ & $0.13 \pm 0.175$ & $\mathbf{0 . 1 4} \pm \mathbf{0 . 1 1 6}$ \\
\cline { 2 - 5 } & 0.4 & $\mathbf{0 . 3 9} \pm 0.208$ & $0.37 \pm 0.12$ & $0.38 \pm \mathbf{0 . 1 1 0}$ \\
\cline { 2 - 5 } & 0.6 & $0.61 \pm 0.226$ & $0.58 \pm 0.115$ & $\mathbf{0 . 6} \pm \mathbf{0 . 1 0 9}$ \\
\cline { 2 - 5 } & 0.8 & $\mathbf{0 . 8} \pm 0.228$ & $0.81 \pm 0.102$ & $\mathbf{0 . 8} \pm \mathbf{0 . 1 0 6}$ \\
\hline
\end{tabular}

TABLE II

BIAS $\left(b^{2} a s^{2}\right)$ OF THREE DIFFERENT ESTIMATORS FOR SELECTED PAIRS $(\rho, H)$ VALUES. BEST RESULTS ARE HIGHLIGHTED IN BOLD.

\begin{tabular}{|c|c|c|c|c|}
\hline \multirow{2}{*}{$\rho$} & \multirow{2}{*}{$\mathrm{H}$} & \multicolumn{3}{|c|}{ Estimators } \\
\cline { 3 - 5 } & DWT & EMD & MEMD \\
\hline \hline \multirow{4}{*}{0} & 0.2 & 0.055 & 0.015 & $\mathbf{0 . 0 0 7}$ \\
\cline { 2 - 5 } & 0.4 & 0.053 & 0.008 & $\mathbf{0 . 0 0 4}$ \\
\cline { 2 - 5 } & 0.6 & 0.054 & 0.006 & $\mathbf{0 . 0 0 4}$ \\
\cline { 2 - 5 } & 0.8 & 0.054 & 0.007 & $\mathbf{0 . 0 0 4}$ \\
\hline \hline \multirow{4}{*}{0.2} & 0.2 & 0.063 & 0.034 & $\mathbf{0 . 0 1 7}$ \\
\cline { 2 - 5 } & 0.4 & 0.056 & 0.024 & $\mathbf{0 . 0 1 2}$ \\
\cline { 2 - 5 } & 0.6 & 0.059 & 0.017 & $\mathbf{0 . 0 0 9}$ \\
\cline { 2 - 5 } & 0.8 & 0.054 & 0.015 & $\mathbf{0 . 0 0 8}$ \\
\hline \hline \multirow{4}{*}{0.8} & 0.2 & 0.059 & 0.034 & $\mathbf{0 . 0 1 6}$ \\
\cline { 2 - 5 } & 0.4 & 0.050 & 0.023 & $\mathbf{0 . 0 1 2}$ \\
\cline { 2 - 5 } & 0.6 & 0.052 & 0.026 & $\mathbf{0 . 0 1 2}$ \\
\cline { 2 - 5 } & 0.8 & 0.052 & 0.023 & $\mathbf{0 . 0 1 1}$ \\
\hline
\end{tabular}

\section{E. Correlograms}

To measure the relationship between pair of channels of each mode, correlogram which a visual representation of the correlation matrix is used. This matrix allows to analyze the link between a pair of channels, and this inter-channel relationship is visualized through a scatter-plot. Thus, all pairs of channels of the $m^{\text {th }}$ IMF are compared using the inter-channels correlation matrix $\operatorname{Corr}_{H, \rho}^{m}$ where each entry $r_{m}^{p, l}=\mathbb{E}\left[c_{m, p}[n] c_{m, l}[n]\right]$ is the correlation coefficient between $c_{m, p}$ and $c_{m, l}, m, l \in\{1,2, \ldots, M\}$ and $p \in\{1,2, \ldots, P\}$. From a practical point of view, $r_{m}^{p, l}$ is estimated by

$$
\hat{r}_{m}^{p, l}=\frac{1}{J} \sum_{j=1}^{J}\left(\frac{1}{N} \sum_{n=1}^{N} c_{m, H_{p}}^{(j)}[n] c_{m, H_{p}}^{(j)}[n]\right)
$$

This value measures the relationship strength between channels $p$ and $l$ of $m^{t h}$ IMF. Results of inter-channel links of each mode reported in figures 9,10 and 11 show that for $\rho \neq 0$, link between channels is evidenced clearly, therefore MEMD conserves the inter-channel correlation of the MfGn process. The new feature is that correlogram falls off with a decrease depending on both $\rho$ and $H$ parameters when going away from the main diagonal, indicating the link degree of interdependence between inter-channels of the IMF. This observation applies to all IMFs. For all $\rho$ values, MEMD produced diagonally dominant correlograms for each IMF. Figure 9 evidences that for uncorrelated channels, regardless $H$ values, correlograms exhibit more pronounced diagonal dominance (without leakage), thus indicating that the channels of the IMFs are also uncorrelated. However, for correlated channels, this dependence on the Hurst exponent is well evidenced on figures 10 and 11. These figures highlight the fact that for channels long-range dependent, coupling between pairs of channels of each IMF is uniform. As the link between channels increases, more important the strength of this uniform coupling between channels of the IMFs is. By contrast, for channels short-range dependent of the input data, the coupling between channels of the modes is non-uniform. This is illustrated by the heterogeneity in terms of intensity of the off-diagonal elements of the correlograms (increased leakage), whose values are also related to the strength of link between pairs of channels. As expected, irrespective of the 

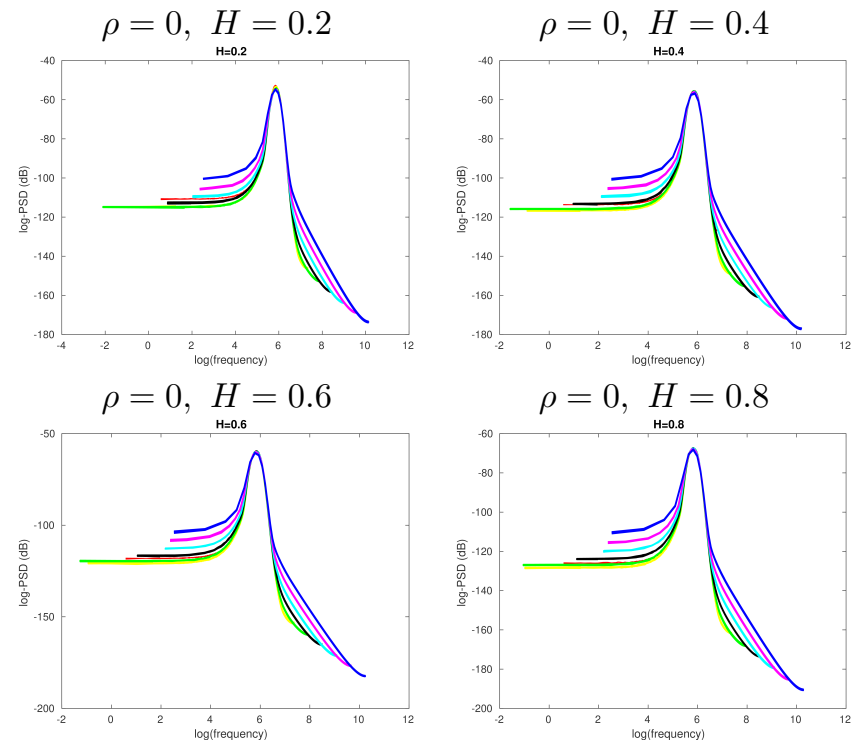

$\rho=0.2, H=0.2$

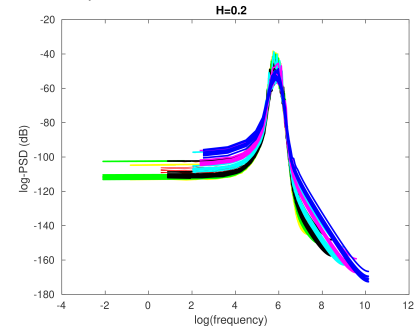

$\rho=0.2, H=0.4$

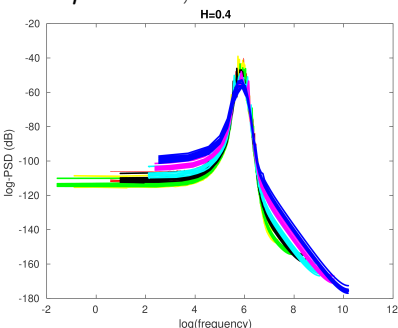

$\rho=0.2, H=0.6$
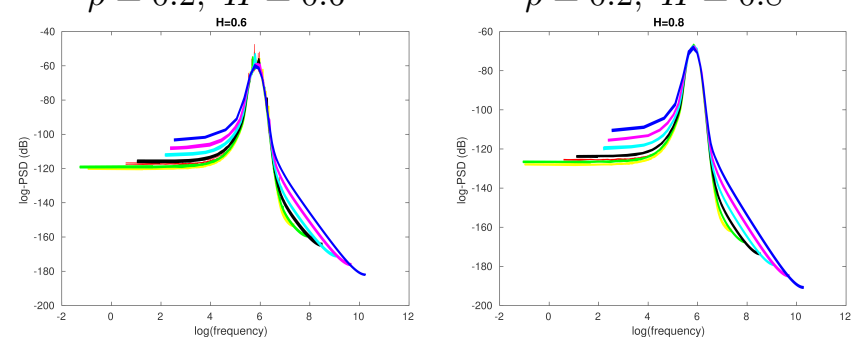

Fig. 5. Normalized PSDs of IMFs for $H \in\{0.2,0.4,0.6,0.8\}$ and $\rho \in$ $\{0,0.2\}$. Overlapping of the frequency bands is obtained using (13).

combinations of pairs $(\rho, H)$ the larger correlations values are along the diagonal. Overall, these results evidence that for correlated channels, MEMD sifts modes that are also correlated and the coupling intensity, observed off-diagonal, is also related to the Hurst exponent value. An increased leakage, in the correlogram, is observed for more fractured channels.

\section{F. Real world turbulent flow data analysis}

Analysis of signals from real world turbulent phenomena is a challenging issue. Filter bank behavior of MEMD is illustrated on near-wall pressure signals of hydrofoil's suction side undergoing a forced rotational motion while facing incoming flow in cavitation tunnel [41]. Pressure measurements are carried out using ten piezo-resistive transducers. Wall pressure signals are expressed in terms of temporal pressure coefficient $C_{p}(t)$, where an example of signal shown in figure 12 can be segmented at least into 5 phases: Laminar flow, Leading
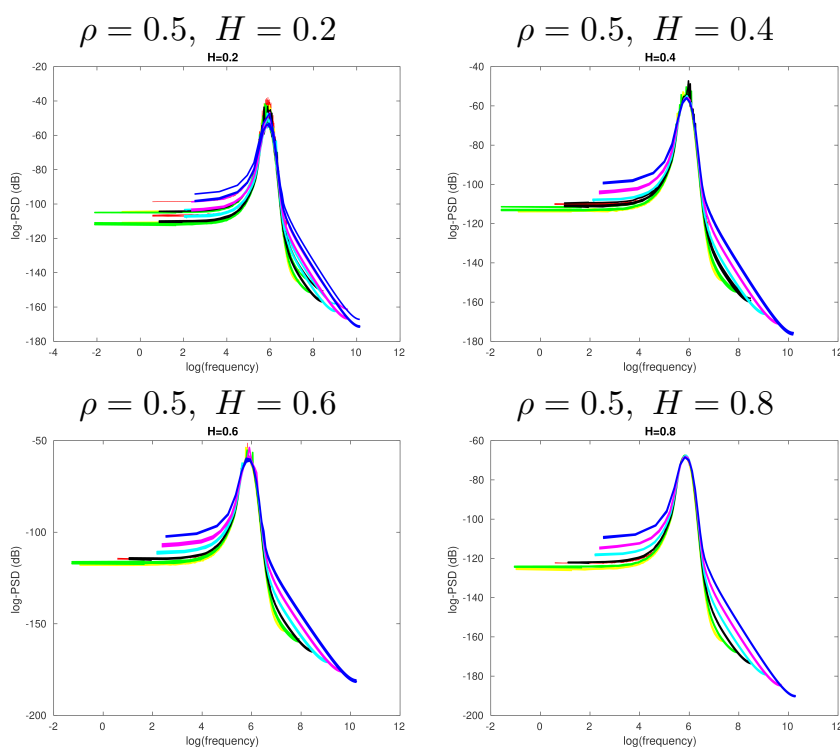

$\rho=0.5, H=0.8$
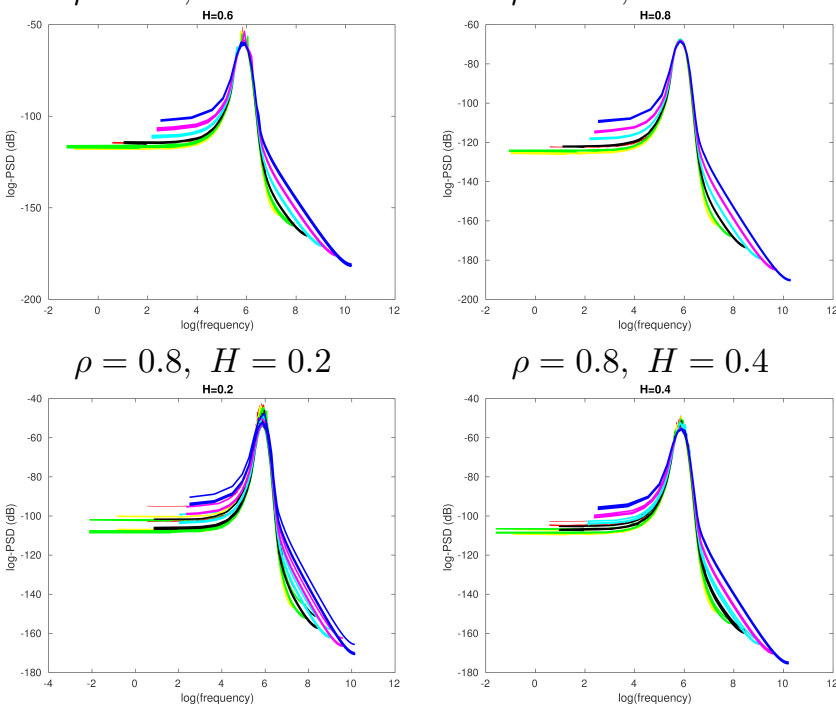

$\rho=0.8, H=0.4$
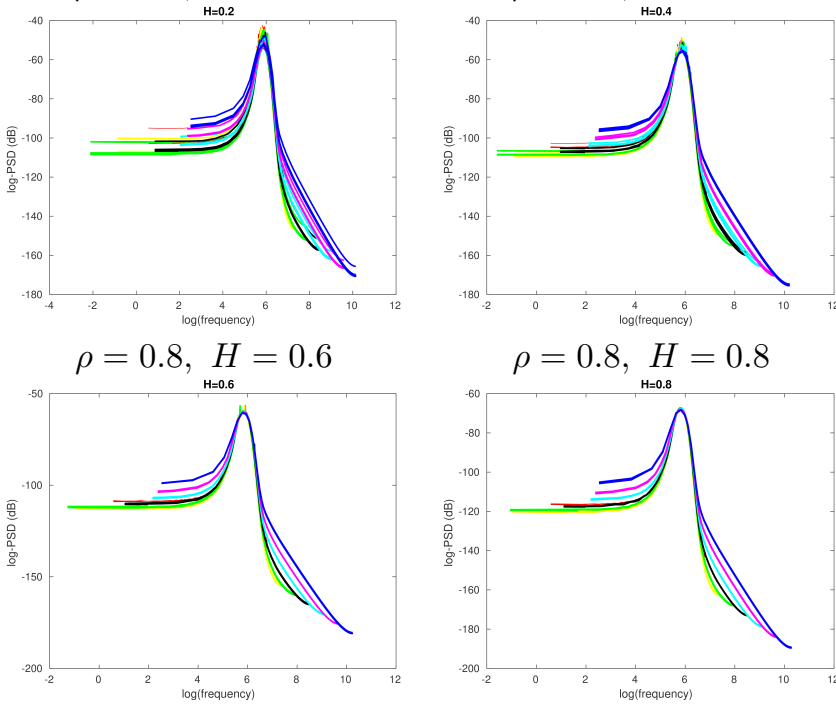

Fig. 6. Normalized PSDs of IMFs for $H \in\{0.2,0.4,0.6,0.8\}$ and $\rho \in$ $\{0.5,0.8\}$. Overlapping of the frequency bands is obtained using (13).
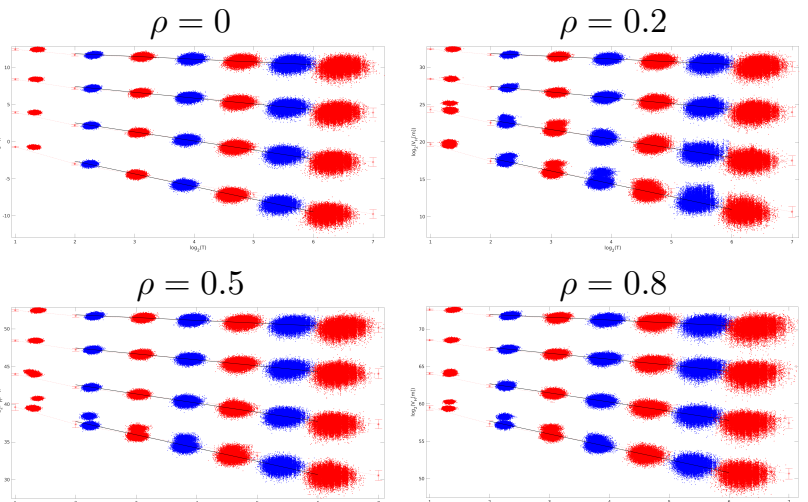

Fig. 7. Relationship between $\log _{2}\left(V_{H_{p}}[m]\right)$ and $\log _{2}\left(\bar{T}_{H_{p}}[m]\right)$ for $H \in$ $\{0.2,0.4,0.6,0.8\}$ and $\rho \in\{0,0.2,0.5,0.8\}$. 

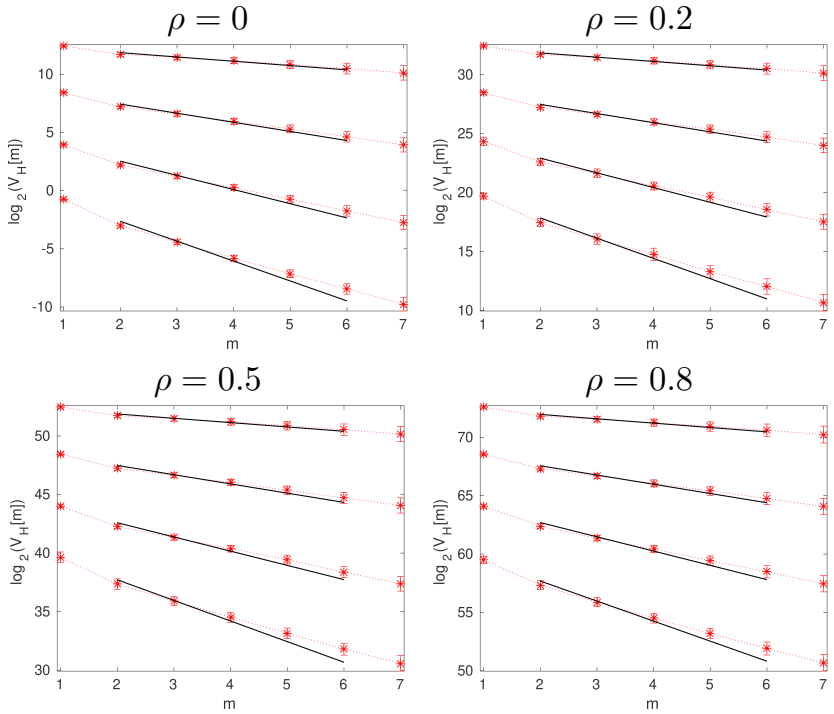

Fig. 8. Relationship between $V_{H_{p}}[m]$ and $m$ indexes for $H \in$ $\{0.2,0.4,0.6,0.8\}$ and $\rho \in\{0,0.2,0.5,0.8\}$.
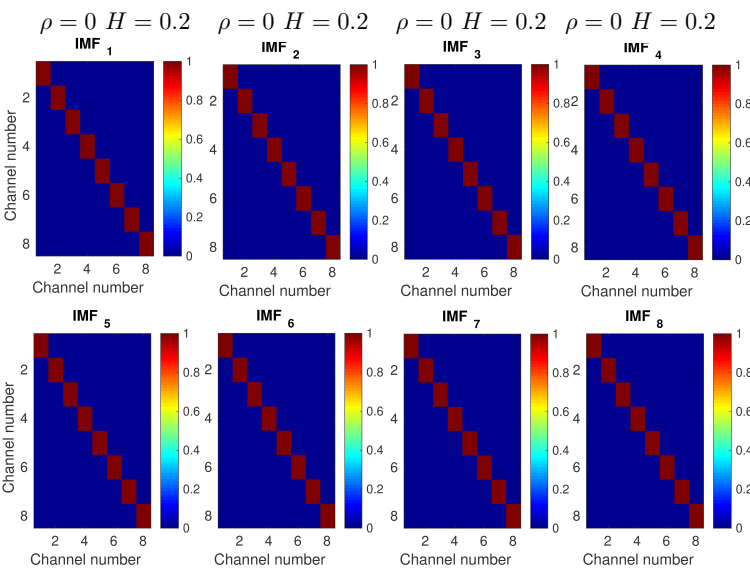

IMF 8
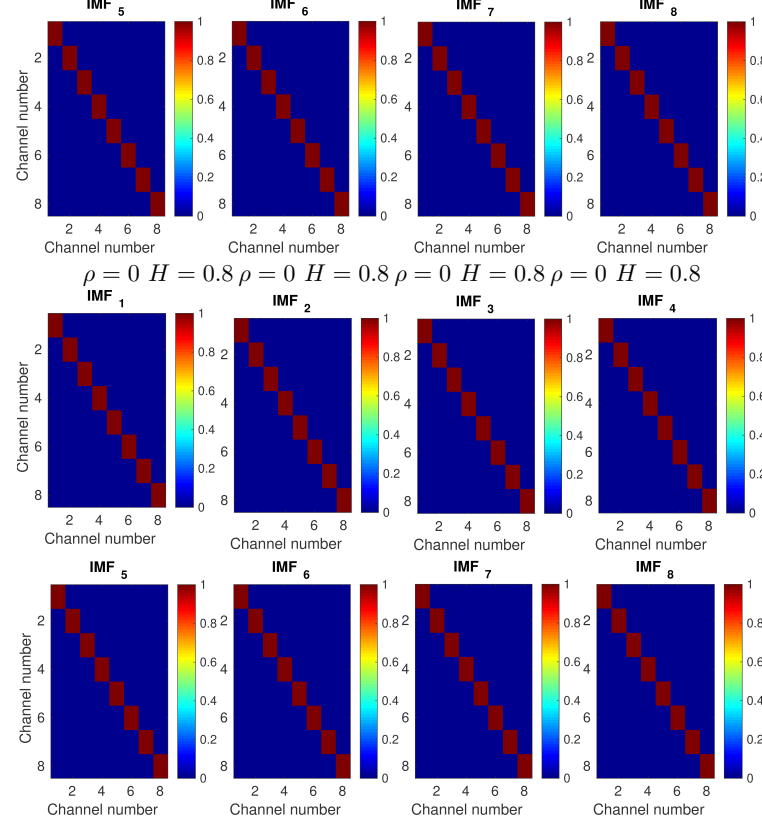

Channel number

$\begin{array}{cccc}2 & 4 & 6 & 8 \\ \text { Channel number }\end{array}$

Channel number

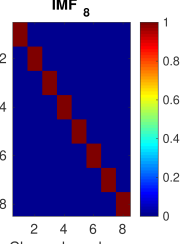

$\begin{array}{cccc}2 & 4 & 6 & 8 \\ \text { Channel number }\end{array}$

Fig. 9. Correlograms of IMFs for $(\rho, H) \in\{(0,0.2),(0,0.8)\}$.

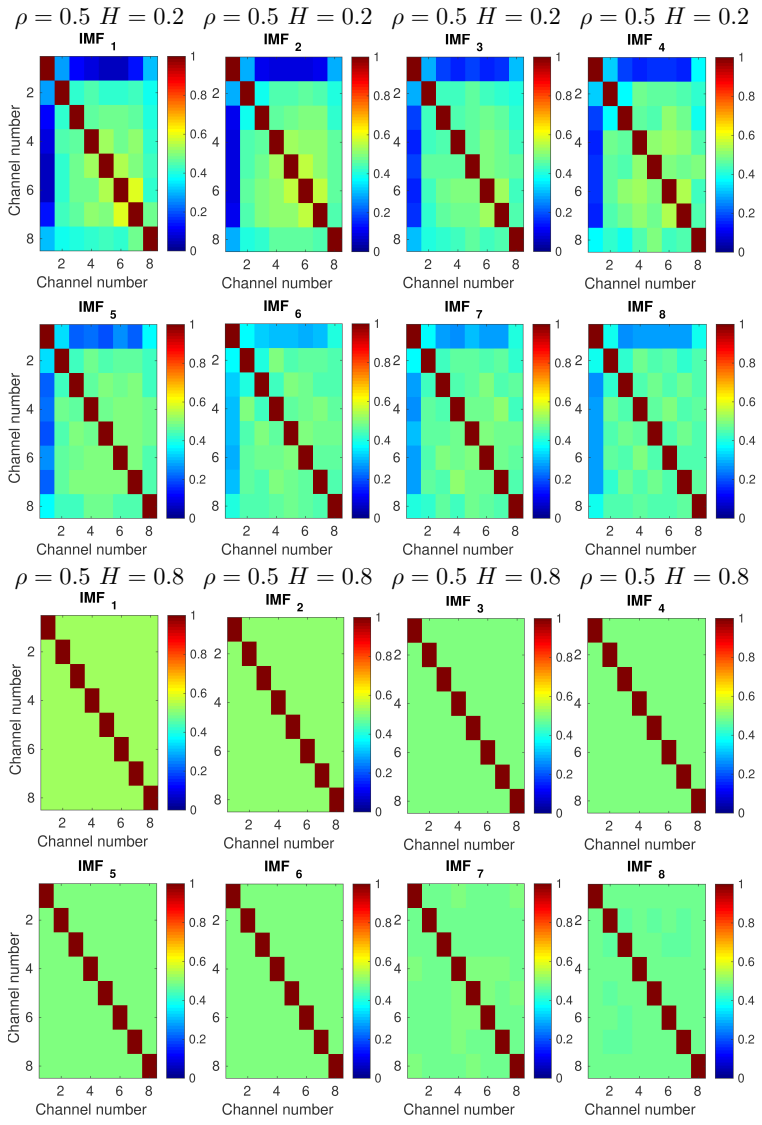

Fig. 10. Correlograms of IMFs for $(\rho, H) \in\{(0.5,0.2),(0.5,0.8)\}$.

edge (LE) separation bubble and transition, turbulent flow, net increasing fluctuations before LE, and separated flow. These phases of the foil show the non-stationarity, randomness and also the complexity of the turbulent flow data. MEMD and EMD (channel-wise) are applied to 10-channels $C_{p}(t)$ signal and the average number of ZCs is calculated for all channels (Figs. 13). As expected EMD failed to effectively align scales from different IMF channels, and this is clearly seen by the dispersion of the dashed blue lines in figure 13(b). While for MEMD, there is the nearly perfect modes alignment (Fig. 13(a)), showing the interdependence between multiple channels of the analyzed pressure signals, as in MfGn process. The calculated slopes of the lines suggest a self-similarity hidden beneath the signal. A LS fitting of the ZCs has been used to estimate the $H$ value $(\hat{H})$ of the data. For MEMD, the slope of the fit line suggests that $\hat{H}=0.95$ (DWT gives $\hat{H}=0.98$ ). This $H$ value, close to 1 , brings out the long-range-dependent nature of the turbulent flow data. PSDs of the sifted modes, by EMD and MEMD shown in figure 14 highlight the fact that the filter bank structure holds for turbulent flow data, and this is more evident for MEMD where the overlapping of the frequency bands associated with the different channels is more prominent compared to EMD. To evaluate the estimation of $H$ value, plotted power spectra of IMFs are superimposed to theoretical PSDs (dashed black line) for $H=0.95$ (Fig. 14). We can observe an almost perfect alignment between the theoretical line and the real 


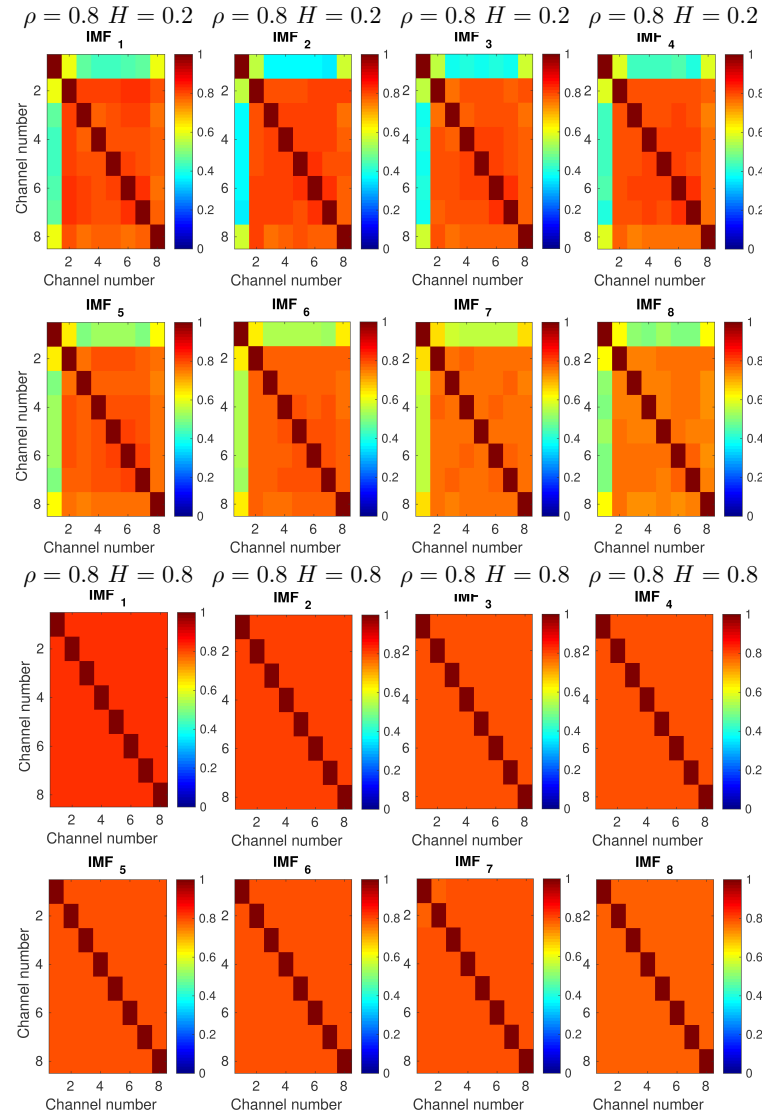

Fig. 11. Correlograms of IMFs for $(\rho, H) \in\{(0.8,0.2),(0.8,0.8)\}$.

data. By transformation (13), we get a very good overlapping of the PSDs which, overall, collapse into a single curve with renormalization. For EMD, PSDs do not present a clear bandpass filters, and this can be seen from the overlapping spectrum in figure 14(b2). We report in figure 15 spectra of IMFs sifted by MEMD with theoretical slopes of pressure spectrum (blue lines). Overall, different power law behaviors are observed over all the frequencies. The estimated slopes, indicated by red lines, are reported in Table III. Using modes selection, we get results very close to the theoretical values reported in [42]. The estimated slope of -2.7 is close to the Kolmogorov's spectral slope $-7 / 3$ for pressure fluctuations. Band-pass IMFs ( $m=2$ to 10$)$ are used for modes selection. Because first IMF (high-pass filter) contains most unwanted very high frequency perturbations, thus it is omitted, and the last IMFs contain unwanted low frequency information. It obvious to see the advantages of using MEMD for the analysis of such signals due its modes alignment ability. MEMD has ability to align common frequency modes across multiple channels in same index IMFs and thus, makes IMF-by-IMF comparisons feasible. For example, by examination of the figure 15, we can associate a "physical phenomenon" to each set of IMFs (which can vary from one to many IMFs). For example, we can associate to the $4^{\text {th }}$ and $5^{\text {th }}$ IMFs the slope $-\frac{7}{3}$, while the IMFs $6-8$ represent the slope -1 .

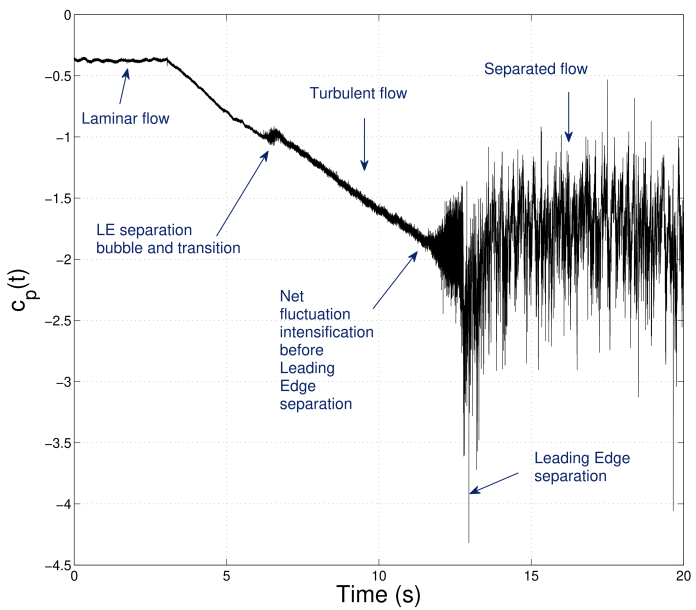

Fig. 12. Pressure signal from transducer \#2 and the associated typical flow.

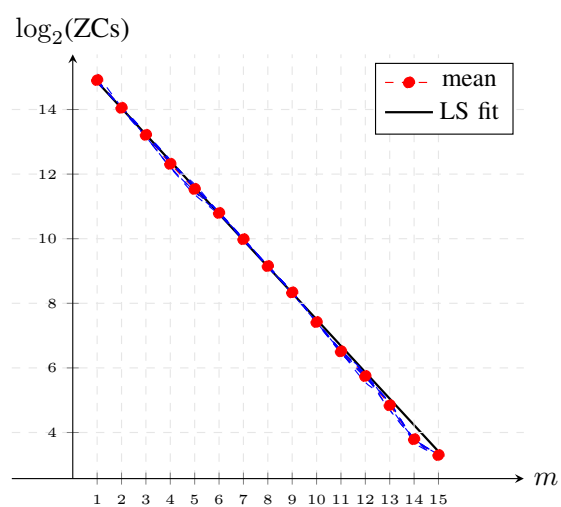

(a)

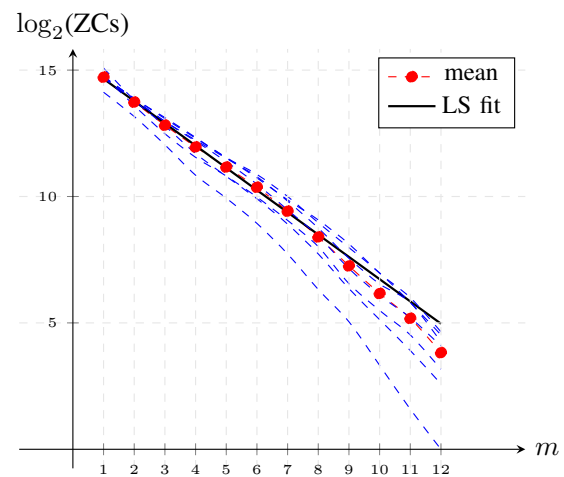

(b)

Fig. 13. Logarithm of $z_{H_{p}}[m]$ versus $m$. Dashed blue lines represents $z_{H_{p}}[m]$ for each channel. Dashed line with red dotes represents the average number of ZCs over all ten channels, and the thick solid black line is the corresponding LS linear fitting for (a) MEMD (b) EMD applied channel-wise.

TABLE III

SLOPES OF THE LINES FOR THE PRESSURE SIGNAL SPECTRUM.

\begin{tabular}{|l|c|c|c|c|}
\hline Theoretical [42] & 2 & -1 & $-7 / 3$ & -6 \\
\hline Measured & 2.7 & -1.3 & -2.7 & -5 \\
\hline
\end{tabular}



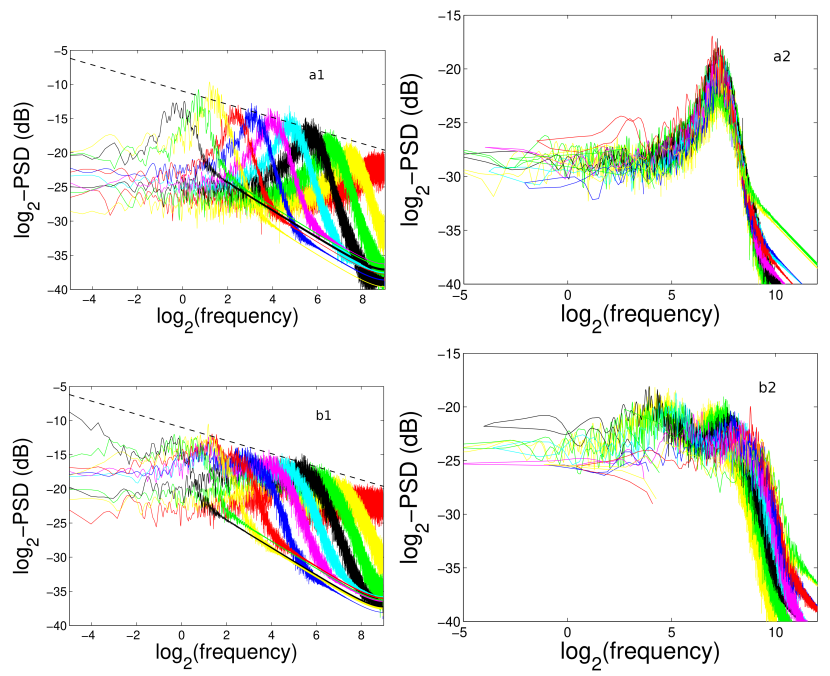

Fig. 14. PSDs of IMFs for MEMD (a1) and EMD (b1). Overlapping of the frequency bands in (a2) and (b2) is obtained using (13) for MEMD and using $\beta_{H} \approx 2$ for EMD [12].

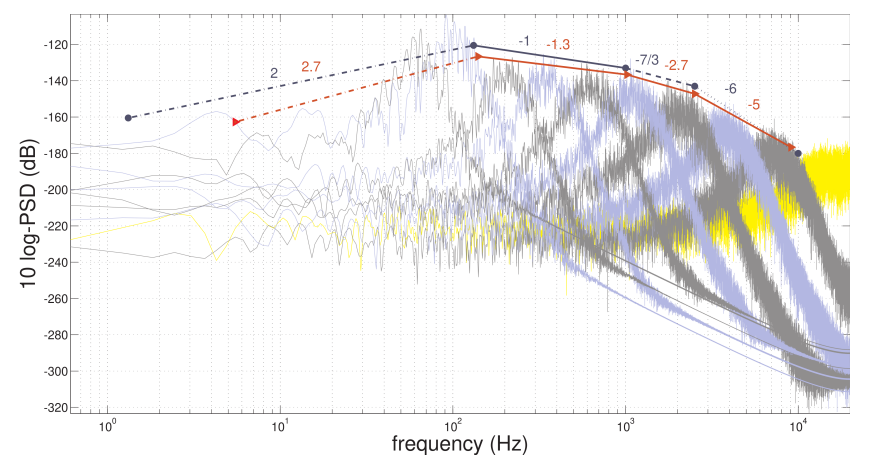

Fig. 15. Pressure signal spectrum.

\section{Detection OF ALPHA RHYTHM IN EEG}

Alpha waves are rhythmic EEG waves occurring at frequency between 8 and $13 \mathrm{~Hz}$, and their detection is a useful indicator of subject's level of stress, concentration or relaxation. MEMD may be an interesting tool to detect such waves using EEG signals which are usually multichannel noisy signals with $1 / f$ property. In this experiment, the aim is to detect when a subject closes his eyes and enters a relaxing mode. This mode usually triggers alpha waves, with frequency ranging from 8 to $13 \mathrm{~Hz}$, and they are most dominant in the occipital region. Signals recorded on three sensors $(\mathrm{O} 1, \mathrm{Oz}$ and $\mathrm{O} 2)$, usually placed on the occipital area are used (Fig. 16). Two recordings, one with "eyes opened" and the other with "eyes closed". The associated signals and their averaged PSD are shown in figures 17(a)-(b). It is expected to have a peak in the band $8-13 \mathrm{~Hz}$ for the black signal (the closed eye signal), due to the Alpha rhythm that appears when the subject closes his eyes. However this is not the case due to dominance of low frequency noise. EEG signal is decomposed using MEMD followed by a filtering (partial reconstruction by excluding the first two modes) and a detrending (removing the residual). Averaged PSD of processed signal reported in figure 17(c), shows the presence of the peak located near the $10 \mathrm{~Hz}$ for

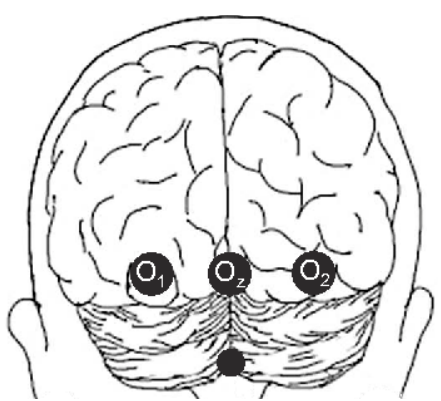

Fig. 16. Occipital scalp electrode locations using the 10-20 IS. The sensors are from the channels $\mathrm{O} 1, \mathrm{Oz}$ and $\mathrm{O} 2$.

signal with "closed eyes". As expected from the behavior analysis of MEMD under MfGn, this application confirms that MEMD operates on multivariate data which exhibit $1 / f$ property. Combined with a modes filtering-detrending strategy, this illustration shows that MEMD has the ability to enhance the detection of frequency of interest such that of Alpha waves.

\section{CONCLUSION}

The aim of this work is to better understanding the way MEMD decomposes MfGn, filling somehow non-existing theoretical framework and the application of an appealing technique to real-world situations. We reported results showing the interest of direct processing of MfGn with MEMD and applications to real turbulent flow data and EEG data. Behavior analysis under MfGn and conclusions drawn are based on extensive simulations with different Hurst exponents and degrees of correlation between pairs of components of this process. Reported findings evidence that MEMD acts as filter bank, on each channel, on both MfGn and real world turbulent flow data. This equivalent filter bank structure is dyadic with constant-Q band-pass filters. For simulated MfGn processes, this filter banks behavior is achieved whatever $\rho$ and $H$ values. Regardless of $\rho$ values, and for both MfGns longrange dependent and short-range dependent, first IMF presents the characteristics of a high-pass filter, while IMFs of higher indexes behave similarly to a band-pass filter. Findings of our work generalize those reported in [15],[18],[20],[19] where behavior was limited to fGn with uncorrelated channels. The study reveals that for correlated channels, MEMD generates modes also with correlated channels, and intensity of interdependence between these channels depends on both $\rho$ and $H$ values of channels of input signal. Intensity of this link needs to be further refined in order to highlight in which proportion the influence of each parameter. The new estimator of Hurst exponent based on MEMD has been illustrated on simulated fGn paths and real data, and the obtained results compared to those based on EMD and DWT based estimators. MEMDestimator performs similarly than DWT estimator but with the lowest standard deviations. Estimated Hurst exponent of real turbulent flow data brings out their long-range-dependent nature. Finally, different power law behaviors are observed on spectra of the modes, and particularly Kolmogorov's spectral slope, showing the potential of MEMD as an exploratory tool 

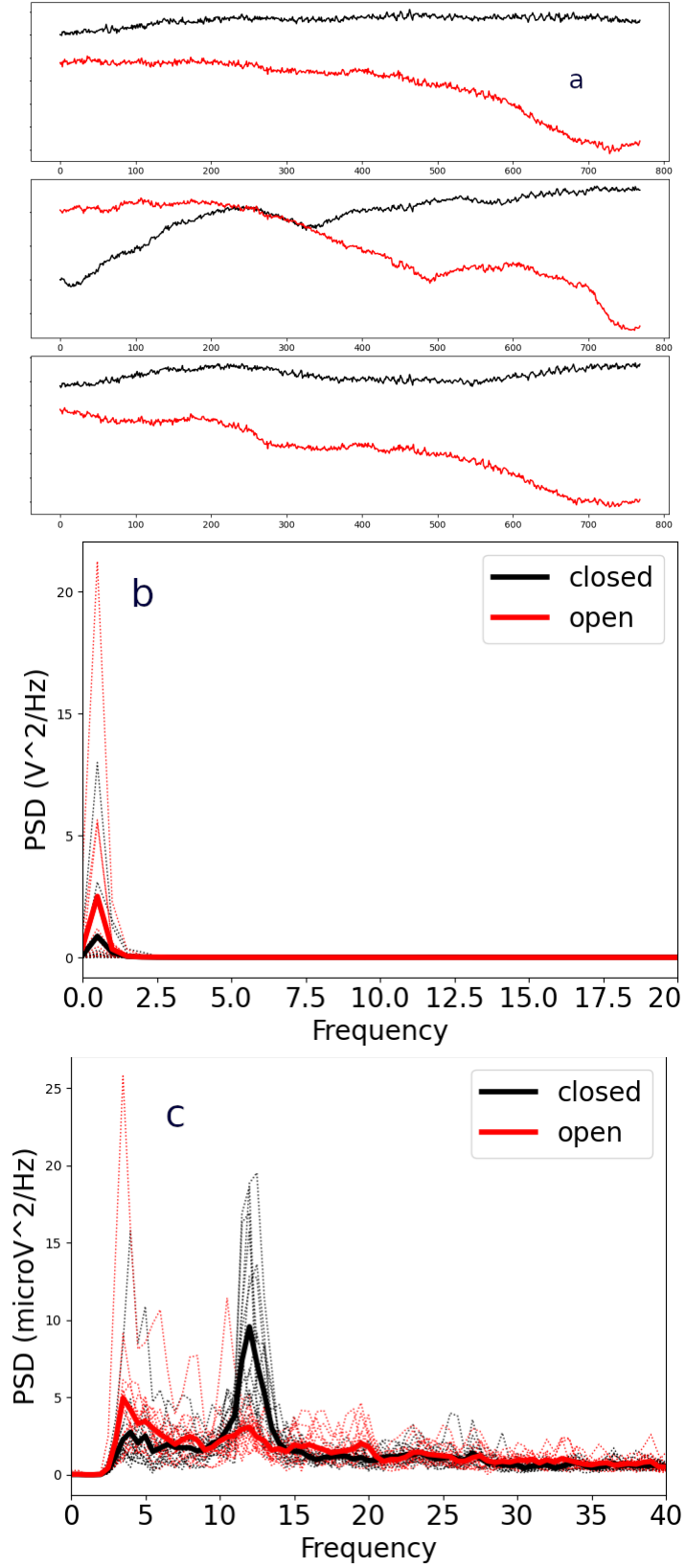

Fig. 17. Average Welch PSD (a) of noisy EEG signals at $\mathrm{O} 1, \mathrm{Oz}$ and $\mathrm{O} 2$, across 5 trials (b). Average Welch PSD of EEG signals at $\mathrm{O} 1, \mathrm{Oz}$ and $\mathrm{O} 2$, across 5 trials after detrending and filtering. The peak around $10 \mathrm{~Hz}$ refers to the alpha wave (c).

for real data. As future work, we plan to extend the present study to the case of less regular models involving a mixture of broadband and narrowband contributions. Estimate $\hat{H}_{p}$ is closely related to slope $\kappa_{H_{p}}$ which in turn is calculated by the setting $\beta_{H_{p}}=2$. Values of $\beta_{H_{p}}$ can be refined to get better results of $\hat{H}_{p}$. Accuracy of $\beta_{H_{p}}$, or that of quadratic relation (Eq. 11) are slightly dependent on the way multivariate IMFs are actually sifted, and particularly on the choice of stopping criterion and uniformity sampling used in Algorithm 1. Further studies are necessary to make this point more precise, and for confirming the obtained estimation results.

\section{REFERENCES}

[1] N. Huang, Z. Shen, S. Long, M. Wu, H. Shih, Q. Zheng, N. Yen, C. Tung, and H. Liu, "The empirical mode decomposition and Hilbert spectrum for nonlinear and non-stationary time series analysis," Proc. R. Soc. A, vol. 454, no. 1971, pp. 903-995, 1998.

[2] J. Cexus and A.O. Boudraa, "Nonstationary signals analysis by TeagerHuang Transform (THT)," in Proc. EUSIPCO, 2006, pp. 1-5.

[3] J.C. Cexus, A.O. Boudraa, A. Bouchikhi, and A. Khenchaf, "Analyce des échos de cibles sonar par transformation de huang-teager (THT)," Traitement du Signal, vol. 24, no. 1, pp. 119-129, 2008.

[4] A. Bouchikhi, A.O. Boudraa, J.C. Cexus, and Th. Chonavel, "Analysis of multicomponent LFM signals by a combined Teager-Huang-Hough Transform," EEE TAES, vol. 50, no. 2, pp. 1222-1233, 2014.

[5] N.E. Huang and S.S.P. Shen, Hilbert-Huang Transform and its Applications. World Scientific, 2014

[6] A.O. Boudraa and J.C. Cexus, "EMD-based signal filtering," IEEE TIM, vol. 56, no. 6, pp. 2196-2202, 2007.

[7] A. Komaty, A.O. Boudraa, B. Augier, and D. Dare, "EMD-based filtering using similarity measure between probablity density functions of IMFs," IEEE TIM, vol. 63, no. 1, pp. 27-34, 2014.

[8] K. Khaldi, A.O. Boudraa, B. Torresani, and Th. Chonavel, "HHT-based audio coding," SIVP, vol. 9, no. 1, pp. 107-115, 2015.

[9] K. Khaldi, A.O. Boudraa, and M. Turki, "Voiced/unvoiced speech classification-based adpative filtering of decomposed empirical modes for speech denoising," IET SP, vol. 10, no. 1, pp. 69-80, 2016.

[10] K. Khaldi, A.O. Boudraa, and A. Komaty, "Speech enhancement using empirical mode decomposition and Teager-Kaiser energy operator," JASA, vol. 135, no. 1, pp. 451-459, 2014.

[11] K. Bensafia, A. Mansour, A.O. Boudraa, S. Haddab, Ph. Ariès, and B. Clement, "Blind separation of ECG signals from noisy signals affected by electrosurgical artifacts," Analog. Integr Circ. Sig. Proc., vol. 104, pp. 191-204, 2020.

[12] P. Flandrin, P. Gonçalvès, and G. Rilling, "Empirical mode decomposition as a filter bank," IEEE SPL, vol. 11, no. 2, pp. 112-114, 2004.

[13] S. Benramdane, J.C. Cexus, A.O. Boudraa, and J.A. Astolfi, "Timefrequency analysis of pressure fluctuations on a hydrofoil undergoing transient pitching motion using Huang-Hilbert and Huang-Teager transforms," in Proc. ASME, 2007, pp. 1-9.

[14] N.U. Rehman and D. Mandic, "Multivariate empirical mode decomposition," Proc. R. Soc. A, vol. 466, no. 2117, pp. 1291-1302, 2010.

[15] — "Filter bank property of multivariate empirical mode decomposition," IEEE TSP, vol. 59, no. 5, pp. 2421-2426, 2011.

[16] A. Komaty, A.O. Boudraa, J.P. Nolan, and D. Dare, "On the behavior of EMD and MEMD in presence of symmetric alpha-stable noise," IEEE $S P L$, vol. 22, no. 7, pp. 818-822, 2015.

[17] D. Mandic, N. Rehman, W. Zhaohua, and N.E. Huang, "Empirical mode decomposition-based time-frequency analysis of multivariate signals: The power of adaptive data analysis," IEEE SPM, vol. 30, no. 6, pp. 74-86, 2013.

[18] A. Komaty, "Traitement et analyse des processus stochastiques par EMD et ses extensions," Ph.D. dissertation, Univ. Brest Occidentale, 2014.

[19] H. Hao, H. Wang, N.U. Rehman, and H. Tian, "A study of the characteristics of MEMD for fractional Gaussian noise," IEICE Trans. Fundamentals, vol. E99-A, pp. 1228-1232, 2016.

[20] A. Komaty and A.O. Boudraa, "Sur l'aspect banc de filtres de l'EMD multivariée," in Colloque GRETSI, 2015, pp. 1-4.

[21] Z. Wu and N.E. Huang, "A study of the characteristics of white noise using the empirical mode decomposition method," Proc. R. Soc. A, vol. 460, pp. 1597-1611, 2003.

[22] J.L. Starck and F. Murtagh, Astronomical Image and Data Analysis. Springer, Berlin, Heidelberg, 2002.

[23] P.O. Amblard and J.F. Coeurjolly, "Identification of the multivariate fractional Brownian motion," IEEE TSP, vol. 59, no. 11, pp. 5152-5168, 2011.

[24] T. Tanaka and D. P. Mandic, "Complex empirical mode decomposition," IEEE SPL, vol. 14, no. 2, pp. 936-939, 2007.

[25] M.U.B. Altaf, T. Gautama, T. Tanaka, and D.P. Mandic, "Rotation invariant complex empirical mode decomposition," in Proc. ICASSP, vol. 3, 2007, pp. 1009-1012.

[26] G. Rilling, P. Flandrin, P. Gonçalvès, and J.M. Lilly, "Bivariate empirical mode decomposition," IEEE SPL, vol. 14, no. 12, pp. 101-104, 2007.

[27] N.U Rehman and D.P. Mandic, "Empirical mode decomposition for trivariate signals," IEEE TSP, vol. 58, no. 3, pp. 1059-1068, 2010.

[28] J. Cui and W. Freeden, "Equidistribution on the sphere," SIAM J. Sci. Comput., vol. 18, no. 2, pp. 595-609, 1997. 
[29] N.U. Rehman, C. Park, N.E. Huang, and D. Mandic, "EMD via MEMD multivariate noise-aided computation of standard EMD," Adv. Adapt Data Anal., vol. 5, no. 2, pp. 1-25, 2013.

[30] B. Mandelbrot and J.W. Van Ness, "Fractional Brownian motions, fractional noises and applications," SIAM review, vol. 10, no. 4, pp. 422-437, 1968.

[31] M. Taqqu, "Fractional Brownian motion and long-range dependence," in Theory and Applications of Long-Range Dependence: Theory and Applications, P. Doukhan, G. Oppenheim, and M.S. Taqqu, Eds. Boston, MA: Birkhauser, 1993, pp. 5-38.

[32] S. Achard, R. Salvador, B. Whitcher, J. Suckling, and E. Bullmore, "A resilient, low-frequency, small-world human brain functional network with highly connected association cortical hubs," J. Neurosc., vol. 16, no. 1, pp. 63-72, 2006.

[33] P.O. Amblard, J.F. Coeurjolly, F. Lavancier, and A. Philippe, "Basic properties of the multivariate fractional Brownian motion," in Bulletin Soc. Math. France, Séminaires et Congrés, no. 28, 2012, pp. 65-87.

[34] J.F. Coeurjolly, P.O. Amblard, and S. Achard, "On multivariate fractional Brownian motion and multivariate fractional Gaussian noise," in Proc. EUSIPCO, 2010, pp. 75-79.

[35] A.O. Boudraa, K. Khaldi, Th. Chonavel, M. Turki Hadj-Alouane, and A. Komaty, "Audio coding via EMD," DSP, vol. 14, pp. 1-11, 2020.

[36] K. Khaldi and A.O. Boudraa, "On signals compression by EMD," IEE Elec. Lett., vol. 48, no. 21, pp. 1329-1331, 2012.

[37] _ " "Audio watermarking via EMD," IEEE TASLP, vol. 21, no. 3, pp. 675-682, 2013.

[38] P. Flandrin, P. Gonçalvès, and G. Rilling, "Emd equivalent filter banks, from interpretation to applications," in Hilbert-Huang Transform and its Applications, N.E. Huang and S.S.P. Shen, Eds. World Scientific Publishing Company, 2005, ch. 3, pp. 57-74.

[39] G. Rilling, P. Flandrin, and P. Gonçalvès, "Empirical mode decomposition, fractional Gaussian noise and Hurst exponent estimation," in Proc. ICASSP, vol. 4, 2005, pp. 489-492.

[40] D. Veitch and P. Abry, "A wavelet-based joint estimator of the parameters of long-range dependence," IEEE TIT, vol. 45, no. 3, pp. 878-897, 1999.

[41] J. Leroux, J. Astolfi, and J. Billard, "An experimental study of unsteady partial cavitation," J. Fluids Eng., vol. 126, no. 1, pp. 94-101, 2004.

[42] W. Bonness, D. Capone, and S. Hambric, "Low wavenumber turbulent boundary layer wall pressure measurements from vibration data on a cylinder in pipe flow," J. Sound Vib., vol. 329, no. 4, pp. 4166-4180, 2010.

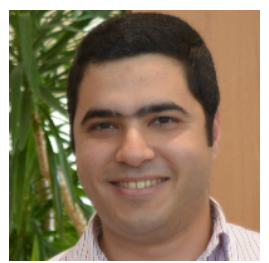

Ali Komaty received his B.E.E degree from the Lebanese University in 2009, M.Sc. degree in signal processing from University of Brest, France in 2010 and the Ph.D. degree in signal processing from the Naval Academy Research Institute, Brest, France, in 2014. He is currently an assistant professor at the University of Sciences and arts of Lebanon, Beirut, Lebanon. His current research interests include speaker recognition, speaker diarization, signal processing and deep neural networks.

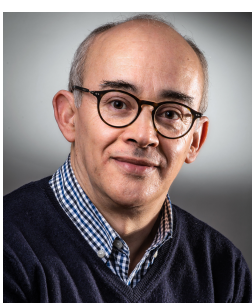

Abdel-Ouahab Boudraa (SM'06) received the B.Sc. degree in Physics from University Constantine, and the Engineer degree in Electronics from Educatel (Liege, Belgium). He also received the M.Sc. degree in Biomedical Engineering from INSA, Lyon, University degree in Nuclear Magnetic Resonance, the Ph.D. degree in Image Processing and the University degrees in Statistics and Modeling and in Positron Emission Tomography all from the University of Lyon 1, France. He received the HdR (accreditation to supervise research) from University of Paris-Saclay, Paris. He is currently full Professor of Electrical Engineering at Ecole Navale/Arts \& Metiers Institute of Technology, Brest, France. His current research interests include time-frequency, AM-FM modeling, Teager-Kaiser energy operators, Data driven methods, Graph signal processing, anomaly detection in time series, transient detection, automated target recognition, passive under-water acoustics and data fusion. Dr. Boudraa was corecipient of the Varian Prize 2003, the Norman Miller Award 2019 and was named Chevalier (knight) in the Ordre des Palmes Académiques 2018.

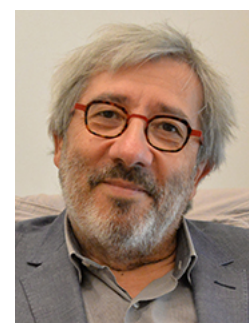

Patrick Flandrin (M'85-SM'01-F'02) received the engineer degree from ICPI Lyon, France, in 1978, and the Doct.-Ing. and Docteur d'état degrees from INP Grenoble, France, in 1982 and 1987, respectively. He joined CNRS in 1982, where he is currently Research Director. Since 1991, he has been with the Signals, Systems and Physics Group, within the Physics Department at ENS de Lyon, France. From 2009 to 2019, he has been President of GRETSI, the French Association for Signal and Image Processing. His research interests include mainly nonstationary signal processing (with emphasis on time-frequency and time-scale methods), scaling stochastic processes and complex systems. He published over 300 research papers and authored two monographs in those areas. Dr. Flandrin was awarded the Philip Morris Scientific Prize in Mathematics (1991), the SPIE Wavelet Pioneer Award (2001), the Prix Michel Monpetit from the French Academy of Sciences (2001), the Silver Medal from CNRS (2010) and the Technical Achievement Award from the IEEE Signal Processing Society (2017). Past Distinguished Lecturer of the IEEE Signal Processing Society (2010-2011), he is a Fellow of the IEEE (2002) and of EURASIP (2009). Elected member of the French Academy of Sciences in 2010, he is its President for the period 2021-2022.

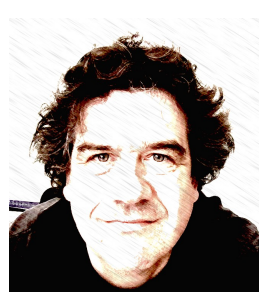

Pierre-Olivier Amblard Dr. Pierre-Olivier Amblard was born in Dijon, France, in 1967. He received the Ingénieur degree in electrical engineering in 1990 from Ecole Nationale Supérieure des Ingénieurs Electriciens de Grenoble, Institut National Polytechnique de Grenoble. He received the DEA (MSc) in signal processing and the Doctorat $(\mathrm{PhD})$ in 1990 and 1994 respectively, both from the INPG. He received the Habilitation à Diriger des Recherches in 2001. Since 1994, he works for the Centre National de la Recherche Scientifique (CNRS) where he is a Directeur de Recherche. He is with GIPSAlab, Grenoble, France, in the team GAIA (Geometry, Learning, Information and Algorithms). From 2010 to 2013, he was in secondment in the Department of Mathematics and Statistics of the University of Melbourne. His research interests include statistical signal processing, non-stationary signal processing, applications of signal processing in physics and neuroscience.

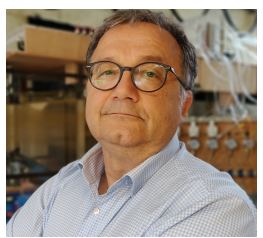

Jacques-Andre Astolfi received his $\mathrm{PhD}$ degree in fluid mechanics from the Institute of Fluid Mechanics of the University of Aix-Marseille II, France in 1990. He received the HdR degree (French National Accreditation to supervise research) from Ecole Centrale de Nantes, University of Nantes in 2000. He was assistant professor from 1991-1996 at the University of Aix-Marseille. Then he was associate professor at Ecole Navale/Arts \& Metiers Institute of Technology up to 2016. He is currently full professor in Fluid Dynamics. He develops experimental research activities in applied hydrodynamics related to naval applications including fluid-structure interaction in complex flows. He is currently interested in high-fidelity data measurements and analysis based on new data-driven modal decomposition for model reduction. He is currently member of the scientific committee of International Symposium on Marine Propeller. $\mathrm{He}$ supervised about twenty-five Phd students and he published about fifty peerreviewed academic papers in leading journals and international conferences. $\mathrm{He}$ is the director of the Research Institute of the French Naval Academy. 